\title{
On the multiplication of spherical functions of reductive spherical pairs of type A
}

\author{
Paolo Bravi and Jacopo Gandini
}

Abstract. Let $G$ be a simple complex algebraic group, and let $K \subset G$ be a reductive subgroup such that the coordinate ring of $G / K$ is a multiplicity-free $G$-module. We consider the $G$-algebra structure of $\mathbb{C}[G / K]$ and study the decomposition into irreducible summands of the product of irreducible $G$-submodules in $\mathbb{C}[G / K]$. When the spherical roots of $G / K$ generate a root system of type $A$, we propose a conjectural decomposition rule, which relies on a conjecture of Stanley on the multiplication of Jack symmetric functions. With the exception of one case, we show that the rule holds true whenever the root system generated by the spherical roots of $G / K$ is a direct sum of subsystems of rank 1 .

\section{Introduction}

Let $G$ be a connected semisimple complex algebraic group, and let $K \subset G$ be a reductive subgroup. Recall that the affine variety $G / K$ is called spherical if it contains an open orbit under a Borel subgroup of $G$. If this is the case, we will also say that $K$ is a spherical subgroup, and that $(G, K)$ is a reductive spherical pair. A fundamental example is that of the symmetric varieties, namely when $K$ is the set of fixed points of an algebraic involution of $G$.

By a result of Vinberg and Kimelfeld [34], reductive spherical subgroups are characterized by the property that the coordinate ring $\mathbb{C}[G / K]$ is a multiplicity-free $G$-module (that is, every isotypic component is irreducible).

Fix a maximal torus $T \subset G$ and a Borel subgroup of $G$ containing $T$, and let $X(T)^{+}$ be the monoid of dominant weights of $G$. For $\lambda \in X(T)^{+}$, we denote by $V(\lambda)$ the irreducible $G$-module of highest weight $\lambda$.

Suppose now that $X$ is an affine spherical homogeneous $G$-variety. Being multiplicity-free, the $G$-module structure of the coordinate ring of $X$ is completely captured by the associated weight monoid, that is, the submonoid $\Lambda_{X}^{+} \subset X(T)^{+}$formed by the highest weights of $\mathbb{C}[X]$. Such monoid is well understood, and can be combinatorially described (see, e.g., [8]). On the other hand, the description of the $G$-algebra structure of $\mathbb{C}[X]$ is still a widely open problem.

Received by the editors June 9, 2021; revised October 28, 2021; accepted November 29, 2021.

Published online on Cambridge Core December 9, 2021.

AMS subject classification: 14M27, 05E10, $20 \mathrm{G} 05$.

Keywords: Reductive spherical pairs, multiplicity-free actions, coordinate rings of spherical varieties, Jack polynomials. 
To be more precise, for $\lambda \in \Lambda_{X}^{+}$, denote by $E_{X}(\lambda) \simeq V(\lambda)$ the irreducible component in $\mathbb{C}[X]$ of highest weight $\lambda$. Then, we have the decomposition

$$
\mathbb{C}[X]=\bigoplus_{\lambda \in \Lambda_{X}^{+}} E_{X}(\lambda) .
$$

Given $\lambda, \mu \in \Lambda_{X}^{+}$, let $E_{X}(\lambda) \cdot E_{X}(\mu)$ denote the submodule of $\mathbb{C}[X]$ generated by the products $f g$ with $f \in E_{X}(\lambda)$ and $g \in E_{X}(\mu)$. We will deal with the following problem: for which $v \in \Lambda_{X}^{+}$, does $E_{X}(v)$ appear inside $E_{X}(\lambda) \cdot E_{X}(\mu)$ ?

Clearly, there are some bounds on the range of weights which can occur inside $E_{X}(\lambda) \cdot E_{X}(\mu)$. For instance, if $v$ is such a weight, then $V(v)$ must appear as a submodule inside the tensor product $V(\lambda) \otimes V(\mu)$.

A more subtle bound comes from the spherical roots of $X$. Let indeed $\mathcal{M}_{X}^{\mathrm{n}} \subset \mathcal{X}(T)$ be the submonoid generated by the set of differences

$$
\left\{\lambda+\mu-v \mid \lambda, \mu, v \in \Lambda_{X}^{+} \text {and } E_{X}(v) \subset E_{X}(\lambda) \cdot E_{X}(\mu)\right\} .
$$

By a fundamental theorem of Knop [18], together with a recent result of Avdeev and Cupit-Foutou [2], the monoid $\mathcal{M}_{X}^{\mathrm{n}}$ is free. Let $\Delta_{X}^{\mathrm{n}}$ be the set of free generators of $\mathcal{M}_{X}^{\mathrm{n}}$, called the spherical roots of $X$ (or, more precisely, the $\mathrm{n}$-spherical roots, in the terminology of Section 4). Then, $\Delta_{X}^{\mathrm{n}}$ is the base of a root system $\Phi_{X}^{\mathrm{n}}$, whose rank is called the rank of $X$. This root system, together with its Weyl group, encodes important information on the geometry of $X$ and of its equivariant embeddings. When $X$ is a symmetric variety, the root system $\Phi_{X}^{\mathrm{n}}$ is essentially the restricted root system attached to $X$.

Similarly to the weight monoid $\Lambda_{X}^{+}$, the set of spherical roots $\Delta_{X}^{\mathrm{n}}$ is also well understood (see, e.g., [8]). In the general context of spherical varieties, both the weight monoid and the spherical roots are a fundamental part of the combinatorial invariants attached by Luna to a spherical variety in his seminal paper [26].

Going back to the problem of decomposing the product of irreducible G-modules inside $\mathbb{C}[X]$, by the very definition of the spherical roots, we see the implication

$$
E_{X}(v) \subset E_{X}(\lambda) \cdot E_{X}(\mu) \Longrightarrow\left\{\begin{array}{c}
V(v) \subset V(\lambda) \otimes V(\mu), \\
\lambda+\mu-v \in \mathbb{N} \Delta_{X}^{\mathrm{n}} .
\end{array}\right.
$$

In several cases, the condition on the right-hand side seems, indeed, close to give a characterization of the irreducible constituents of $E_{X}(\lambda) \cdot E_{X}(\mu)$. However, there are counterexamples (see, e.g., Example 6.4); thus, we are led to consider other possible descriptions.

In particular, in the present paper, we will focus on the case of the affine spherical homogeneous spaces $X=G / K$ for a simple group $G$, whose spherical roots form a root system of type $A$. The classification of the reductive spherical subgroups for a simple group was first obtained by Krämer [23], and more recently in arbitrary characteristic by Knop and Röhrle [19]. For a complete list of the cases we will deal with, see the table at the end of Section 3 (for the symmetric cases) and the table at the beginning of Section 6 (for the non-symmetric cases).

When $X$ is a symmetric variety with restricted root system of type $A$, a general conjectural rule for decomposing the product of two irreducible components of $\mathbb{C}[X]$ 
follows from a long-standing conjecture of Stanley [33] on the multiplication of Jack symmetric functions (see Conjecture 3.2). Such conjectural description holds true when the rank of $X$ is 1 , as a consequence of a Pieri rule for the multiplication of Jack symmetric functions proved by Stanley himself [33].

The connection between Stanley's context and ours stems from the well-known fact that specializing the Jack symmetric functions to a suitable parameter defined by $X$ (which is essentially the multiplicity of the restricted roots) and setting the extra variables to 0 yields indeed a basis for the space $\mathbb{C}[X]^{K}$ of the spherical functions on $X$. This point of view was already exploited by Graham and Hunziker [16], who dealt with a problem similar to the one considered in this paper in the case of the symmetric varieties with restricted root system of type $A$.

Our main contribution with the present paper is to show how a general conjectural rule for decomposing the product of two irreducible constituents in $\mathbb{C}[X]$ also follows from Stanley's mentioned conjecture for all the nonsymmetric affine spherical homogeneous varieties whose spherical roots generate a root system of type $A$. When this root system is a direct sum of subsystems of rank 1 , which happens in most cases, we will see (with the exception of one case) that such description holds true thanks to Stanley's Pieri rule for Jack symmetric functions.

To state the mentioned decomposition rule, we will introduce a different normalization for the spherical roots of $X$ and will define a slight variation of $\Phi_{X}^{\mathrm{n}}$, where we replace some of the spherical roots of $X$ with their half. More precisely, we will associate to $X$ a based root datum $\mathcal{R}_{X}$ (see Proposition 4.3 for its definition), having the same Weyl group of $\Phi_{X}^{\mathrm{n}}$ and with the property that the weight monoid $\Lambda_{X}^{+}$can be naturally regarded as a submonoid of the dominant weights of $\mathcal{R}_{X}$.

Let $G_{X}$ be the complex reductive group associated to $\mathcal{R}_{X}$, and for $\lambda \in \Lambda_{X}^{+}$, denote by $V_{X}(\lambda)$ the irreducible $G_{X}$-module associated to $\lambda$ (regarded as a dominant weight for $G_{X}$ ). Then, we have the following conjecture (see Conjecture 5.1).

Conjecture 1.1 Let $X$ be an affine spherical homogeneous variety for a simple group $G$ such that $\Phi_{X}^{\mathrm{n}}$ is of type A. Let $\lambda, \mu, v \in \Lambda_{X}^{+}$, then

$$
E_{X}(v) \subset E_{X}(\lambda) \cdot E_{X}(\mu) \Longleftrightarrow\left\{\begin{array}{c}
V_{X}(v) \subset V_{X}(\lambda) \otimes V_{X}(\mu), \\
\lambda+\mu-v \in \mathbb{N} \Delta_{X}^{\mathrm{n}} .
\end{array}\right.
$$

Our main theorem is the following (see Propositions 5.8 and 5.12 and Corollary 5.10).

Theorem 1.2 Let $X$ be an affine spherical homogeneous variety for a simple group $G$. Suppose that $\Phi_{X}^{\mathrm{n}}$ is of type $\mathrm{A}$, and that $X \neq \mathrm{F}_{4} / \operatorname{Spin}(9)$.

(i) If $\Phi_{X}^{\mathrm{n}}$ is a direct sum of rank 1 subsystems, then Conjecture 1.1 holds for $X$.

(ii) If Stanley's conjecture on the multiplication of Jack symmetric functions holds true, then Conjecture 1.1 holds true for X.

In the case of $F_{4} / \operatorname{Spin}(9)$, which remains excluded from the previous theorem, Conjecture 1.1 is based on computational experiments.

The strategy that we will adopt to prove the previous theorem is somehow homogeneous the various cases that we will consider. Indeed, we will show that in all cases (with the exception of $\mathrm{F}_{4} / \mathrm{Spin}(9)$, and three other cases that can be easily reduced 
to some other case), we can find a reductive overgroup $\widehat{G} \supset G$ with a symmetric $\widehat{G}$ variety $Y$ and a reductive subgroup $H \subset G$ with a symmetric $H$-variety $Z$, respectively, endowed with a $G$-equivariant isomorphism and with an $H$-equivariant embedding

$$
Z \hookrightarrow X \stackrel{\sim}{\longrightarrow} Y .
$$

Both the symmetric varieties $Y$ and $Z$ will have a restricted root system of type $A$, and we will see that the previous maps induce an isogeny from the based root datum $\mathcal{R}_{X}$ to the direct sum $\mathcal{R}_{Y} \oplus \mathcal{R}_{Z}$ (see Proposition 5.6).

This will allow us to show that the validity of Conjecture 1.1 in the various cases follows from the analogous statement for the symmetric varieties with restricted root system of type A, which in turn follows from Stanley's conjecture, and from Stanley's Pieri rule when $\Phi_{X}^{\mathrm{n}}$ is a direct sum of rank 1 root subsystems.

The fact that the $G$-action on $X$ can be extended to a $\widehat{G}$-action making $X$ into a symmetric $\widehat{G}$-variety will be basically a consequence of a suitable factorization of $\widehat{G}$ as a product of two reductive subgroups (one of them being $G$, and the other one a symmetric subgroup of $\widehat{G}$ with the desired property). Such factorizations were first classified by Onishchik [29], and in arbitrary characteristic by Liebeck, Saxl, and Seitz [24].

In the last section, we will also show that the same technique described above can also be applied in the case of the spherical variety $\mathrm{SO}(2 n+1) / \mathrm{GL}(n)$ (whose spherical root system is not of type $A$ ), to decompose its root datum into the direct sum of those associated with two symmetric varieties of Hermitian type (see Theorem 6.1). This leads us to consider the decomposition problem also for another class of reductive spherical pairs $(G, K)$, namely when $K$ is a spherical Levi subgroup of $G$, in which case $\Phi_{G / K}^{\mathrm{n}}$ is of type B or C. Here, the problem seems quite unexplored, even in the symmetric case. We show that the case of the spherical Levi subgroups reduces to that of the symmetric Levi subgroups (that is, to the case of the symmetric varieties of Hermitian type), and we formulate a conjectural rule for the decomposition of the product of two irreducible components in $\mathbb{C}[G / K]$ in this case as well (see Conjecture $6.2)$.

\section{Notation and preliminaries}

If $H$ is a connected algebraic group, we will denote by $\mathcal{X}(H)$ its character lattice. If $H$ acts on a vector space $V$, we will denote by $V^{H}$ the invariant subspace and by $V^{(H)}$ the semi-invariant subset, that is, the union of the semi-invariant subspaces

$$
V_{\chi}^{(H)}=\{v \in V \mid h \cdot v=\chi(h) v \quad \forall h \in H\}
$$

with $\chi \in X(H)$. If $\Xi$ is a lattice, we will denote by $\Xi^{\vee}$ its dual lattice, namely $\Xi^{\vee}=$ $\operatorname{Hom}_{\mathbb{Z}}(\Xi, \mathbb{Z})$.

Let $G$ be a semisimple complex algebraic group. If $K \subset G$, we will denote by $\mathrm{N}_{G}(K)$ the normalizer of $K$ in $G$, and by $Z_{G}(K)$ the centralizer of $K$ in $G$. Fix a maximal torus $T \subset G$ and a Borel subgroup $B$ containing $T$. We denote by $\Phi$ the root system of $G$ associated to $T$, and by $\Delta$ the base defined by $B$ and by $\Phi=\Phi^{+} \sqcup \Phi^{-}$the corresponding decomposition into positive and negative roots. When $\Phi$ is an irreducible root system 
of rank $n$, we will order the set of simple roots $\Delta=\left\{\alpha_{1}, \ldots, \alpha_{n}\right\}$ following Bourbaki's notation.

The monoid of dominant weights of $G$ will be denoted by $X(T)^{+}$. Given $\lambda \in X(T)^{+}$, the irreducible representation of $G$ of highest weight $\lambda$ will be denoted by $V_{G}(\lambda)$, or simply by $V(\lambda)$ when no ambiguity is possible.

Let $G / K$ be an affine spherical variety. Equivalently, $K$ is a reductive group, and the coordinate ring $\mathbb{C}[G / K]$ is multiplicity-free as a $G$-module. By the multiplicity-free property, the description of the $G$-module structure of $\mathbb{C}[G / K]$ is equivalent to the description of the monoid of the spherical weights

$$
\Lambda_{G / K}^{+}=\left\{\lambda \in X(T)^{+} \mid V(\lambda)^{K} \neq 0\right\} .
$$

Because $K$ is reductive, notice that $V(\lambda)$ admits a nonzero $K$-invariant vector if and only if the same holds for its dual $V(\lambda)^{*}$. Therefore, with this notation, we have the decomposition

$$
\mathbb{C}[G / K]=\mathbb{C}[G]^{K} \simeq \bigoplus_{\lambda \in \mathcal{X}(T)^{+}} V(\lambda)^{*} \otimes V(\lambda)^{K} \simeq \bigoplus_{\lambda \in \Lambda_{G / K}^{+}} V(\lambda) .
$$

For every $\lambda \in \Lambda_{G / K}^{+}$, we denote by $E_{G / K}(\lambda) \simeq V(\lambda)$ the corresponding isotypic (irreducible) component in $\mathbb{C}[G / K]$.

The previous description can be generalized from the realm of $K$-invariant functions to that of $K$-semi-invariant functions, with respect to the right action of $G$ on itself. Indeed, for every character $\chi \in X(K)$, the eigenspace $\mathbb{C}[G]_{\chi}^{(K)}$ is also a multiplicity-free $G$-module. Inside $\mathbb{C}[G]_{\chi}^{(K)}$, every $\lambda \in \Lambda^{+}$with $\left(V(\lambda)^{*}\right)_{\chi}^{(K)} \neq 0$ yields an isotypic component $E_{G / K}(\lambda, \chi) \simeq V(\lambda)$. In this setting, the relevant weight monoid is that of the quasi-spherical weights

$$
\Omega_{G / K}^{+}=\left\{\lambda \in X(T)^{+} \mid V(\lambda)^{(K)} \neq 0\right\} .
$$

The algebra structure of the invariant ring $\mathbb{C}[G]^{K}$ is encoded by the associated spherical functions. For every $\lambda \in \Lambda_{G / K}^{+}$, fix a nonzero element $f_{\lambda} \in E_{G / K}(\lambda)^{K}$. The $K$-invariant space in $V(\lambda)$ is one-dimensional; thus, the spherical functions $\left\{f_{\lambda}\right\}_{\lambda \in \Lambda_{G / K}^{+}}$form a basis for the space of $K$-invariant functions

$$
\mathbb{C}[G]^{K \times K}=\bigoplus E_{G / K}(\lambda)^{K} .
$$

More explicitly, if $\lambda \in \Lambda_{G / K}^{+}$, the spherical function $f_{\lambda}$ can be constructed (up to a scalar factor) as a matrix coefficient by taking nonzero $K$-invariants vectors $v_{\lambda} \in V(\lambda)$ and $\psi_{\lambda} \in V(\lambda)^{*}$ and setting

$$
f_{\lambda}(g K)=\left\langle\psi_{\lambda}, g \cdot v_{\lambda}\right\rangle .
$$

The decomposition of the product of irreducible $G$-submodules inside $\mathbb{C}[G / K]$ is encoded in the structure constants for the multiplication of the corresponding spherical functions (see [31, Theorem 3.2]).

Proposition 2.1 If $\lambda, \mu, v \in \Lambda_{G / K}^{+}$and if $f_{\lambda} f_{\mu}=\sum a_{\lambda, \mu}^{v} f_{v}$, then

$$
E_{G / K}(v) \subset E_{G / K}(\lambda) \cdot E_{G / K}(\mu) \Longleftrightarrow a_{\lambda, \mu}^{v} \neq 0 .
$$


More generally, a similar characterization can be given in terms of the support of the tensor product of the $K$-semi-invariant vectors of the quasi-spherical representations (see [9, Lemma 19], [4, Lemma 1.2]).

Proposition 2.2 Let $\lambda, \mu, v \in \Omega_{G / K}^{+}$, and let $\chi, \chi^{\prime} \in X(K)$ be such that the semiinvariant subspaces $V(\lambda)_{\chi}^{(K)}$ and $V(\mu)_{\chi^{\prime}}^{(K)}$ are nonzero. Let $v_{\lambda} \in V(\lambda)_{\chi}^{(K)}$ and $v_{\mu} \in$ $V(\mu)_{\chi^{\prime}}^{(K)}$ be nonzero, and let $\pi_{v}: V(\lambda) \otimes V(\mu) \rightarrow V[v]$ denote the projection onto the isotypic component $V[v] \subset V(\lambda) \otimes V(\mu)$ of highest weight $v$. Then,

$$
E_{G / K}\left(\nu, \chi+\chi^{\prime}\right) \subset E_{G / K}(\lambda, \chi) \cdot E_{G / K}\left(\mu, \chi^{\prime}\right) \Longleftrightarrow \pi_{v}\left(v_{\lambda} \otimes v_{\mu}\right) \neq 0 .
$$

\section{Multiplication of spherical functions of symmetric pairs of type A}

Let $G$ be semisimple and simply connected, and let $\vartheta: G \rightarrow G$ be an algebraic involution. We denote by $K=G^{9}$ the set of fixed points. Then, $K$ is a connected reductive subgroup of $G$, which has finite index in $\mathrm{N}_{G}(K)$.

Let $A \subset G$ be a maximal split torus, that is, $\vartheta(a)=a^{-1}$, for all $a \in A$, and $A$ is maximal with this property. Fix a maximal torus $T$ containing $A$, then $\vartheta(T)=T$. Let us denote by the same letter $\vartheta$ the induced involution on $t^{*}$, then $\vartheta(\Phi)=\Phi$ and the Killing form is preserved by $\vartheta$. We also choose the set of positive roots and the Borel subgroup $B \supset T$ in such a way that $\vartheta(\alpha) \in \Phi^{-}$whenever $\alpha \in \Phi^{+}$and $\alpha \neq \vartheta(\alpha)$ (see [11, Lemma 1.2]).

Denote $X=G / K$, and let $A_{X} \simeq A / A \cap K$ be the image of $A$ in $X$. Notice that

$$
A \cap K=\left\{a \in A \mid a^{2}=1\right\} ;
$$

thus, $X\left(A_{X}\right)=2 X(A)$ is identified with the lattice

$$
\Lambda_{X}=\{\chi-\vartheta(\chi) \mid \chi \in \mathcal{X}(T)\} .
$$

Define

$$
\widetilde{\Phi}_{X}=\{\alpha-\vartheta(\alpha) \mid \alpha \in \Phi, \alpha \neq \vartheta(\alpha)\}
$$

the set of restricted roots of $X$. Then, $\widetilde{\Phi}_{X}$ is a possibly nonreduced) root system in $\Lambda_{X} \otimes \mathbb{R} \simeq \mathcal{X}(A) \otimes \mathbb{R}$, with base

$$
\widetilde{\Delta}_{X}=\{\alpha-\vartheta(\alpha) \mid \alpha \in \Delta, \vartheta(\alpha) \neq \alpha\}
$$

and with Weyl group

$$
W_{X} \simeq \mathrm{N}_{G}(A) / \mathrm{Z}_{G}(A) \simeq \mathrm{N}_{K}(A) / \mathrm{Z}_{K}(A) .
$$

The weight lattice and the root lattice of $\widetilde{\Phi}_{X}$ are, respectively, identified with $\Lambda_{X} \simeq X\left(A_{X}\right)$ and with $X\left(A / \mathrm{N}_{A}(K)\right) \subset X\left(A_{X}\right)$ (see [36, Lemmas 2.3 and 3.1]). Finally, the monoid of the spherical weights $\Lambda_{X}^{+}$is obtained by intersecting the monoid of the dominant weights with the sublattice of $X(T)$ defined by $X\left(A_{X}\right)$ (see [35, Théorème 3]):

$$
\Lambda_{X}^{+}=\Lambda_{X} \cap \mathcal{X}(T)^{+}
$$


By a result of Richardson [30, Corollary 11.5], the restriction of functions yields an algebra isomorphism

$$
\mathbb{C}[X]^{K} \stackrel{\sim}{\longrightarrow} \mathbb{C}\left[A_{X}\right]^{W_{X}} .
$$

Up to normalization, the restriction of the spherical function $f_{\lambda}$ to $A_{X}$ is a specialization of the Jacobi polynomial $P_{\lambda}^{(k)}$ associated with the root system $\widetilde{\Phi}_{X}$ (see [17, Section 8.4]). More precisely, let $m=\left(m_{\widetilde{\alpha}}\right)$ be the multiplicity function of the restricted roots, that is, for $\widetilde{\alpha} \in \widetilde{\Phi}_{X}$,

$$
m_{\widetilde{\alpha}}=|\{\beta \in \Phi \mid \beta-\vartheta(\beta)=\widetilde{\alpha}\}| .
$$

Then, up to a scalar factor, the restriction $f_{\lambda_{\mid A_{X}}}$ coincides with the specialized Jacobi polynomial $P_{\lambda}^{(m / 2)}$.

Therefore, the decomposition of the products of the irreducible $G$-modules inside $\mathbb{C}[X]$ is encoded in the structure constants for the multiplication of the corresponding specialized Jacobi polynomials:

$$
\begin{array}{r}
\text { If } \lambda, \mu, v \in \Lambda_{X}^{+} \text {and if } P_{\lambda}^{(m / 2)} P_{\mu}^{(m / 2)}=\sum a_{\lambda, \mu}^{v} P_{v}^{(m / 2)} \text {, then } \\
E_{X}(v) \subset E_{X}(\lambda) \cdot E_{X}(\mu) \Longleftrightarrow a_{\lambda, \mu}^{v} \neq 0 .
\end{array}
$$

Let $\alpha \in \Phi$ be such that $\alpha \neq \vartheta(\alpha)$ : then, either $\vartheta(\alpha)=-\alpha$, or $\alpha$ and $\vartheta(\alpha)$ are orthogonal, or $\left\langle\vartheta(\alpha), \alpha^{\vee}\right\rangle=1$ (in which case, $\alpha-\vartheta(\alpha) \in \Phi^{+}$; see [36, Lemma 2.3]). Thus, if $\sigma \in \widetilde{\Delta}_{X}$, say $\sigma=\alpha-\vartheta(\alpha)$, we define the coroot $\sigma^{\vee} \in \Lambda_{X}^{\vee}$ as follows:

(i) If $\vartheta(\alpha)=-\alpha$, then

$$
\sigma^{\vee}=\frac{1}{2} \alpha_{\mid \Lambda_{X}}^{\vee}
$$

(ii) If $\alpha$ and $\vartheta(\alpha)$ are orthogonal, then

$$
\sigma^{\vee}=\alpha^{\vee} \Lambda_{X} \cdot
$$

(iii) If $\alpha-\vartheta(\alpha) \in \Phi^{+}$, then

$$
\sigma^{\vee}=(\alpha-\vartheta(\alpha))^{\vee}{ }_{\mid \Lambda_{X}}
$$

Notice that the previous definition only depends on $\sigma$, and not on $\alpha$. If indeed $\lambda \in \Lambda_{X}$, then in all cases, we have

$$
\left\langle\lambda, \sigma^{\vee}\right\rangle=\frac{2(\lambda, \alpha-\vartheta(\alpha))}{\|\alpha-\vartheta(\alpha)\|^{2}} .
$$

For later use, we now introduce a slight modification of the restricted root system. Denote

$$
\Delta_{X}^{\dagger}=\left\{\sigma \in \widetilde{\Delta}_{X} \cap 2 \Delta \mid\left\langle\sigma, \tau^{\vee}\right\rangle \in 2 \mathbb{Z} \forall \tau \in \widetilde{\Delta}_{X}\right\}
$$

and define

$$
\Delta_{X}=\left(\widetilde{\Delta}_{X} \backslash \Delta_{X}^{\dagger}\right) \cup \frac{1}{2} \Delta_{X}^{\dagger}
$$

Then, $\Delta_{X}$ is the base of a (reduced) root system $\Phi_{X}$ (see, e.g., [20, Proposition 3.1]): indeed, the scalar product between any two distinct elements in $\Delta_{X}$ remains nonpositive, and the pairing among roots remains integer. Notice that $\Phi_{X}$ has also 
Weyl group $W_{X}$. If moreover $\Delta_{X}$ is irreducible of rank $>1$ and distinct from $\widetilde{\Delta}_{X}$, then $\Delta_{X}$ and $\widetilde{\Delta}_{X}$ have necessarily dual types.

Accordingly, we extend the weight lattice with the normalized restricted roots, and we define $\Xi_{X}$ to be the lattice generated by $\Lambda_{X}$ and $\Delta_{X}$ (namely by $\Lambda_{X}$ and $\frac{1}{2} \Delta_{X}^{\dagger}$ ).

For $\sigma \in \Delta_{X}$, we define the coroot $\sigma^{\vee} \in \Xi_{X}^{\vee}$ similarly as we did before: if $\sigma \in \Phi^{+}$, then $\sigma^{\vee} \in \Xi_{X}^{\vee}$ is the restriction of the associated coroot in $\Lambda^{\vee}$; if instead $\sigma=2 \alpha$ with $\alpha \in \Delta$, then we define $\sigma^{\vee}=\frac{1}{2} \alpha^{\vee} \mid \Xi_{X}$; and finally, if $\sigma=\alpha-\vartheta(\alpha)$ with $\left\langle\vartheta(\alpha), \alpha^{\vee}\right\rangle=0$, then we define $\sigma^{\vee}=\alpha^{\vee} \mid \Xi_{X}$.

Set

$$
\Delta_{X}^{\vee}=\left\{\sigma^{\vee}: \sigma \in \Delta_{X}\right\},
$$

regarded as a subset of the dual lattice $\Xi_{X}^{\vee}$. Then, the triple $\mathcal{R}_{X}=\left(\Xi_{X}, \Delta_{X}, \Delta_{X}^{\vee}\right)$ is a based root datum, in the following (usual) sense.

A based root datum is a triple $\left(\Xi, S, \alpha \mapsto \alpha^{\vee}\right)$ such that:

(i) $\Xi$ is a free abelian group of finite rank, $S$ is a finite subset of $\Xi$, and $\alpha \mapsto \alpha^{\vee}$ is a map from $S$ into $\Xi^{v}$.

(ii) For all $\alpha \in S$, the pairing $\left\langle\alpha, \alpha^{\vee}\right\rangle$ is equal to 2 .

(iii) The group $W$ of automorphisms of $\Xi$ generated by the reflections

$$
s_{\alpha}: \Xi \longrightarrow \Xi, \quad \xi \longmapsto \xi-\left\langle x, \alpha^{\vee}\right\rangle \alpha,
$$

with $\alpha \in S$ is finite.

(iv) The $W$-orbit of $S$ is a reduced root system $R$ in the real subspace of $\Xi_{\mathbb{R}}$ generated by $S$, and $S$ is a base of $R$.

Notice that in our case the root datum $\mathcal{R}_{X}$ is semisimple, namely the base $S$ spans $\Xi_{\mathbb{R}}$ as a vector space.

Let

$$
\Xi_{X}^{+}:=\left\{\lambda \in \Xi_{X}:\left\langle\lambda, \sigma^{\vee}\right\rangle \geqslant 0 \forall \sigma \in \Delta_{X}\right\}
$$

be the monoid of the dominant weights of $\mathcal{R}_{X}$. Then, we have a (possibly strict) inclusion $\Lambda_{X}^{+} \subset \Xi_{X}^{+}$, and $\Xi_{X}^{+}=\Xi_{X} \cap \Lambda^{+}$.

On the weight lattice $\Xi_{X}$, we will consider the partial order $\leqslant_{X}$ defined by $\widetilde{\Delta}_{X}$ as follows:

$$
\pi_{1} \leqslant X \pi_{2} \quad \text { if } \quad \pi_{2}-\pi_{1} \in \mathbb{N} \widetilde{\Delta}_{X}
$$

When $\widetilde{\Delta}_{X}=\Delta_{X}$, this is the usual dominance order on $\Xi_{X}$; otherwise, it is a refinement of the dominance order where the simple roots in $\frac{1}{2} \Delta_{X}^{\dagger}$ are only taken with even coefficients.

\subsection{A conjectural rule when $\Phi_{X}$ is of type $A$}

When $\widetilde{\Phi}_{X}$ (or equivalently $\Phi_{X}$ ) is of type $A$, then up to a normalization, the Jacobi polynomials $P_{\lambda}^{(k)}$ coincide with the Jack symmetric polynomials $J_{\lambda}^{(1 / k)}$ (see, e.g., [3, Proposition 3.3]). Thus, in this case, the restriction of the spherical function $f_{\lambda}$ to $A_{X}$ is a scalar multiple of the specialized Jack polynomial $J_{\lambda}^{(2 / m)}$. 
As is well known, when dealing with symmetric polynomials, it is often convenient to consider symmetric functions in infinitely many variables. Here, we do the same, and instead of working with the Jack polynomials, we pass to the Jack symmetric functions $J_{\lambda}^{(k)}$, which form a basis, when $\lambda$ vary among all possible partitions, of the ring of symmetric functions in infinitely many variables with coefficients in $\mathbb{Q}(k)$. As a general reference on the Jack symmetric functions, see [33] and [28, Section VI.10].

We now recall a conjecture of Stanley concerning the multiplication of the Jack symmetric functions.

Conjecture 3.1 [33, Conjecture 8.3] Write $J_{\lambda}^{(k)} J_{\mu}^{(k)}=\sum_{v} \frac{g_{\lambda, \mu}^{v}(k)}{j_{v}(k)} J_{v}^{(k)}$. Then, the function $g_{\lambda, \mu}^{v}(k)$ is a polynomial in $k$, with nonnegative integer coefficients.

To explain the denominator $j_{v}(k)$, recall that the Jack symmetric functions are pairwise orthogonal with respect to the scalar product $\langle,\rangle_{k}$ defined on the basis of the power-sum symmetric functions $p_{\lambda}$ ( $\lambda$ being a partition) as follows:

$$
\left\langle p_{\lambda}, p_{\mu}\right\rangle_{k}=\left\{\begin{array}{cl}
z_{\lambda} k^{\ell(\lambda)} & \text { if } \lambda=\mu, \\
0 & \text { if } \lambda \neq \mu .
\end{array}\right.
$$

Here, $\ell(\lambda)$ denotes the length of $\lambda$, and if $m_{i}$ is the number of parts in $\lambda$ equal to $i$, then

$$
z_{\lambda}=\left(1^{m_{1}} 2^{m_{2}} \cdots\right) m_{1} ! m_{2} ! \cdots
$$

Therefore, expanding the product of two Jack symmetric functions in terms of Jack symmetric functions, one has

$$
J_{\lambda}^{(k)} J_{\mu}^{(k)}=\sum_{v} \frac{\left\langle J_{\lambda}^{(k)} J_{\mu}^{(k)}, J_{v}^{(k)}\right\rangle_{k}}{\left\langle J_{v}^{(k)}, J_{v}^{(k)}\right\rangle_{k}} J_{v}^{(k)} .
$$

Then, $j_{v}(k)=\left\langle J_{v}^{(k)}, J_{v}^{(k)}\right\rangle_{k}$, and $g_{\lambda, \mu}^{v}(k)=\left\langle J_{\lambda}^{(k)} J_{\mu}^{(k)}, J_{v}^{(k)}\right\rangle_{k}$. There is a well-known combinatorial formula which expresses $j_{v}(k)$ as a polynomial in $k$ with nonnegative integer coefficients in terms of hooks of $v$ (see [33, Theorem 5.8]).

It is known that the functions $g_{\lambda, \mu}^{v}(k)$ are polynomials in $k$ with integer coefficients: this follows from a result of Knop and Sahi [20], showing that the coefficients of the expansion of the Jack symmetric functions in terms of the monomial symmetric functions are polynomial in $k$ with nonnegative integer coefficients.

Conjecture 3.1 is known to be true when one of the two partitions $\lambda$ and $\mu$ consists of a single row: this follows from a generalization of the Pieri rule for Jack symmetric functions proved by Stanley himself [33, Theorem 6.1].

Let us come back to a symmetric space $X=G / K$ with restricted root system of type A. If we normalize properly the spherical functions $f_{\lambda}$ and write $f_{\lambda} f_{\mu}=\sum a_{\lambda, \mu}^{v} f_{v}$, then $a_{\lambda, \mu}^{v}=g_{\lambda, \mu}^{v}(2 / m)$. On the other hand, by the very definition of the scalar product $\langle,\rangle_{k}$, specializing the Jack symmetric function $J_{\lambda}^{(k)}$ at $k=1$ yields a nonzero scalar multiple of the Schur function $s_{\lambda}$. Thus, $g_{\lambda, \mu}^{v}(1)$ is nonzero if and only if the LittlewoodRichardson coefficient $c_{\lambda, \mu}^{v}$ associated to $\lambda, \mu, v$ in the restricted root system associated to $G / K$ is nonzero. 
For a symmetric space $X$ with restricted root system of type $A$, we get then

$$
E_{X}(v) \subset E_{X}(\lambda) \cdot E_{X}(\mu) \Longleftrightarrow g_{\lambda, \mu}^{v}(2 / m) \neq 0,
$$

and Stanley's conjecture immediately implies

$$
E_{X}(v) \subset E_{X}(\lambda) \cdot E_{X}(\mu) \Longleftrightarrow c_{\lambda, \mu}^{v} \neq 0 .
$$

When the rank of the restricted root system is 1, only one-row partitions occur as dominant weights; therefore, equation (3.2) holds true thanks to Stanley's generalization of the Pieri rule mentioned above.

To be more explicit, let $G_{X}$ be the connected semisimple group defined by the based root datum $\mathcal{R}_{X}=\left(\Xi_{X}, \Delta_{X}, \Delta_{X}^{\vee}\right)$, and correspondingly, let $T_{X} \subset G_{X}$ and $B_{X} \subset G_{X}$ be a maximal torus and a Borel subgroup containing $T_{X}$ inducing $\mathcal{R}_{X}$. For $\pi \in \Xi_{X}^{+}$(and in particular for $\left.\pi \in \Lambda_{X}^{+}\right)$, let $V_{X}(\pi)$ be the irreducible $G_{X}$-module with highest weight $\pi$. Then, Stanley's Conjecture 3.1 yields the following (weaker) conjecture.

Conjecture 3.2 Let $X$ be a symmetric variety with restricted root system of type $A$, and let $\lambda, \mu, v \in \Lambda_{X}^{+}$. Then,

$$
E_{X}(v) \subset E_{X}(\lambda) \cdot E_{X}(\mu) \Longleftrightarrow\left\{\begin{array}{c}
V_{X}(v) \subset V_{X}(\lambda) \otimes V_{X}(\mu), \\
v \leqslant_{X} \lambda+\mu .
\end{array}\right.
$$

When the restricted root system of $X$ is of type $A_{1}$, the previous conjecture holds true by Stanley's Pieri rule for Jack symmetric functions.

Notice that Conjecture 3.2 is easily shown when $G=H \times H$ and $K=\operatorname{diag}(H)$ for some reductive algebraic group $H$ (even in types other than $\mathrm{A}$ ), in which case $X \simeq H$ regarded as $H \times H$-variety (see, e.g., [10, Lemma 3.4]).

Remark 3.3 Notice that in all cases of Table 1 but case Sym.A1 with $n=2$, it always holds the equality $\Delta_{X}=\widetilde{\Delta}_{X}$. In particular, in all these cases, Conjecture 3.2 follows immediately from (3.2), and the condition $v \leqslant_{X} \lambda+\mu$ is redundant. On the other hand, to deduce Conjecture 3.2 from (3.2) in the case $X=\mathrm{SL}(2) / T$, we need to use the saturation property of the tensor product of SL(2).

Remark 3.4 Rather than $\mathcal{R}_{X}$, in Conjecture 3.2, it would have been more natural to use the based root datum $\widetilde{\mathcal{R}}_{X}=\left(\Lambda_{X}, \widetilde{\Delta}_{X}, \widetilde{\Delta}_{X}^{\vee}\right)$, and define the semisimple group $G_{X}$ accordingly. Indeed, this formulation follows immediately from (3.2), and is also simpler, because the inequality $v \leqslant_{X} \lambda+\mu$ becomes redundant. However, computational experiments suggest that the formulation that we adopted is closer to possible generalizations (see Section 6, where we consider the case of the symmetric subgroups of Hermitian type).

In a slightly different form, Conjecture 3.2 was also considered by Graham and Hunziker [16].

For completeness, we list in Table 1 all the symmetric varieties with a restricted root system of type $A$. In all cases, we also give the simple restricted roots and a minimal set of generators for the weight monoid $\Lambda_{X}^{+}$. 


\begin{tabular}{ccccc}
\hline & $G$ & $K$ & $\Phi_{G / K}$ & $m_{\sigma}$ \\
\hline Sym.A1 & $\operatorname{SL}(n), n \geqslant 2$ & $\operatorname{SO}(n)$ & $\mathrm{A}_{n-1}$ & 1 \\
Sym.A2 & $\operatorname{SL}(n) \times \operatorname{SL}(n), n \geqslant 2$ & $\operatorname{diag}(\operatorname{SL}(n))$ & $\mathrm{A}_{n-1}$ & 2 \\
Sym.A3 & $\operatorname{SL}(2 n), n \geqslant 2$ & $\operatorname{Sp}(2 n)$ & $\mathrm{A}_{n-1}$ & 4 \\
Sym.A4 & $\operatorname{Spin}(n), n \geqslant 5$ & $\operatorname{Spin}(n-1)$ & $\mathrm{A}_{1}$ & $n-2$ \\
Sym.A5 & $\mathrm{E}_{6}$ & $\mathrm{~F}_{4}$ & $\mathrm{~A}_{2}$ & 8 \\
\hline
\end{tabular}

Table 1: Symmetric pairs with restricted root system of type $A_{r}$

(i) $\operatorname{SL}(2) / \operatorname{SO}(2): \Delta_{X}=\left\{\alpha_{1}\right\}, \Lambda_{X}^{+}$is generated by $2 \omega_{1}$;

(ii) $\operatorname{SL}(\mathbf{n}) / \mathrm{SO}(\mathbf{n}), n \geqslant 3: \Delta_{X}=\left\{2 \alpha_{i}: 1 \leqslant i \leqslant n-1\right\}, \Lambda_{X}^{+}$is generated by the weights $2 \omega_{i}$ with $1 \leqslant i \leqslant n-1$

(iii) $(\mathrm{SL}(\mathbf{n}) \times \operatorname{SL}(\mathbf{n})) / \operatorname{diag}(\mathrm{SL}(\mathbf{n})), n \geqslant 2: \Delta_{X}=\left\{\alpha_{i}+\alpha_{i}^{\prime}: 1 \leqslant i \leqslant n-1\right\}, \Lambda_{X}^{+}$is generated by the weights $\omega_{i}+\omega_{i}^{\prime}$ with $1 \leqslant i \leqslant n-1$;

(iv) $\operatorname{SL}(2 \mathbf{n}) / \operatorname{Sp}(2 \mathbf{n}), n \geqslant 2: \Delta_{X}=\left\{\alpha_{2 i-1}+2 \alpha_{2 i}+\alpha_{2 i+1}: 1 \leqslant i \leqslant n-1\right\}, \Lambda_{X}^{+}$is generated by the weights $\omega_{2 i}$ with $1 \leqslant i \leqslant n-1$;

(v) $\operatorname{Spin}(2 \mathbf{n}+1) / \operatorname{Spin}(2 \mathbf{n}), n \geqslant 2: \Delta_{X}=\left\{2\left(\sum_{i=1}^{n} \alpha_{i}\right)\right\}, \Lambda_{X}^{+}$is generated by $\omega_{1}$;

(vi) $\operatorname{Spin}(2 \mathbf{n}) / \operatorname{Spin}(2 \mathbf{n}-1), n \geqslant 3: \Delta_{X}=\left\{2\left(\sum_{i=1}^{n-2} \alpha_{i}\right)+\alpha_{n-1}+\alpha_{n}\right\}, \Lambda_{X}^{+}$is generated by $\omega_{1}$;

(vii) $\mathrm{E}_{6} / \mathrm{F}_{4}: \quad \Delta_{X}=\left\{2 \alpha_{1}+\alpha_{2}+2 \alpha_{3}+2 \alpha_{4}+\alpha_{5}, \alpha_{2}+\alpha_{3}+2 \alpha_{4}+2 \alpha_{5}+2 \alpha_{6}\right\}, \quad \Lambda_{X}^{+} \quad$ is generated by $\omega_{1}$ and $\omega_{6}$.

\section{Root systems associated to a spherical homogeneous space}

We now recall how to attach a based root system (or more precisely, three based root systems) to a spherical homogeneous space $G / K$, which for the purposes of the present paper we can assume affine. General references for these constructions are $[7,18]$.

Let $\Lambda_{G / K}$ be the weight lattice of $G / K$, defined as

$$
\Lambda_{G / K}=\left\{\chi \in X(T) \mid \mathbb{C}(G / K)_{\chi}^{(B)} \neq 0\right\} .
$$

Because $K$ is reductive, notice that $\Lambda_{G / K}$ is generated as a lattice by $\Lambda_{G / K}^{+}$. In all the cases that we will consider, we will actually have $\Lambda_{G / K}^{+}=\Lambda_{G / K} \cap \Lambda^{+}$.

Looking at the multiplication of spherical modules, we can associate three root systems to $G / K$. For a fixed $G / K$, the three root systems will have the same Weyl group $W_{G / K}$ : what changes is only the root normalization.

- The n-spherical roots of $G / K$. The first root system, that we denote by $\Phi_{G / K}^{\mathrm{n}}$, is defined by considering the multiplication of spherical modules inside the invariant space $\mathbb{C}[G]^{K}$. If $\lambda, \mu, v \in \Lambda_{G / K}^{+}$is a triple of spherical weights such that $E(v) \subset$ $E(\lambda) \cdot E(\mu)$, then $\lambda+\mu-v \in \Lambda_{G / K} \cap \mathbb{N} \Delta$. Denote by $\mathcal{M}_{G / K}^{\mathrm{n}}$ the monoid generated by 
all possible differences $\lambda+\mu-v \in \Lambda_{G / K}$ with $\lambda, \mu, v \in \Lambda_{G / K}^{+}$as above. This is a free monoid (see [18, Theorem 1.3], [2, Theorem 4.11]). Its (unique) set of free generators, denoted by $\Delta_{G / K}^{\mathrm{n}}$, is the set of the n-spherical roots of $G / K$. It is the base of a reduced root system, $\Phi_{G / K}^{\mathrm{n}}$ (see [18, Theorem 1.3]).

- The sc-spherical roots of $G / K$. The second root system, that we denote by $\Phi_{G / K}^{\text {sc }}$, is defined by considering the multiplication of quasi-spherical modules inside the semi-invariant space $\mathbb{C}[G]^{(K)}$. If $\lambda, \mu, v \in \Omega_{G / K}^{+}$is a triple of quasi-spherical weights such that $E_{\chi+\chi^{\prime}}(v) \subset E_{\chi}(\lambda) \cdot E_{\chi^{\prime}}(\mu)$, then $\lambda+\mu-v \in \Lambda_{G / K} \cap \mathbb{N} \Delta$. Let $\mathcal{M}_{G / K}^{\text {sc }}$ be the monoid generated by all possible differences $\lambda+\mu-v$ with $\lambda, \mu, v \in \Omega_{G / K}^{+}$as above. Again, this is a free monoid (see [18, Corollary 7.6], [4, Proposition 5]). Its (unique) set of free generators, denoted by $\Delta_{G / K}^{\mathrm{sc}}$, is the set of the sc-spherical roots of $G / K$. It is the base of a reduced root system, $\Phi_{G / K}^{\text {sc }}$

- The minimal spherical roots of $G / K$. By definition, we have inclusions $\mathcal{M}_{G / K}^{\mathrm{n}} \subset$ $\mathcal{M}_{G / K}^{\mathrm{sc}} \subset \Lambda_{G / K}$. The two root monoids $\mathcal{M}_{G / K}^{\mathrm{n}}$ and $\mathcal{M}_{G / K}^{\mathrm{sc}}$ actually generate the same cone in $\Lambda_{G / K} \otimes_{\mathbb{Z}} \mathbb{Q}$. However, neither the n-spherical roots nor the sc-spherical roots need to be primitive elements inside $\Lambda_{G / K}$ : the set of the minimal spherical roots of $G / K$, denoted by $\Delta_{G / K}^{\min }$, is the set of the primitive elements in $\Lambda_{G / K}$ associated to the extremal rays of the cone generated by $\mathcal{M}_{G / K}^{\mathrm{n}}$ in $\Lambda_{G / K} \otimes_{\mathbb{Z}} \mathbb{Q}$. The minimal spherical roots of $G / K$ form the base of a reduced root system, that we denote by $\Phi_{G / K}^{\min }$.

It is clear from their construction that the three root systems $\Phi_{G / K}^{\mathrm{min}}, \Phi_{G / K}^{\mathrm{sc}}$, and $\Phi_{G / K}^{\mathrm{n}}$ all have the same Weyl group $W_{G / K}$.

If $G / K$ is a symmetric variety, we have $\widetilde{\Delta}_{G / K}=\Delta_{G / K}^{\mathrm{n}}$ (see [18, Theorem 6.7]).

Both the n-spherical roots and the sc-spherical roots of $G / K$ arise as minimal spherical roots of a quotient of $G / K$. Denote indeed by $\bar{K}$ the spherical closure of $K$, defined as the kernel of the action of $\mathrm{N}_{G}(K)$ on $\chi(K)$. Then, both $\mathrm{N}_{G}(K)$ and $\bar{K}$ are spherical subgroups of $G$, which satisfy

$$
\Delta_{G / K}^{\mathrm{n}}=\Delta_{G / \mathrm{N}_{G}(K)}^{\min } \quad \Delta_{G / K}^{\mathrm{sc}}=\Delta_{G / \bar{K}}^{\min } .
$$

This explains our terminology. Moreover, we have equalities

$$
\Lambda_{G / \mathrm{N}_{G}(K)}=\mathbb{Z} \Delta_{G / K}^{\mathrm{n}} \quad \Lambda_{G / \bar{K}}=\mathbb{Z} \Delta_{G / K}^{\mathrm{sc}} .
$$

The inclusion $\mathbb{Z} \Delta_{G / K}^{\min } \subset \Lambda_{G / K}$ is strict in general: it is an equality precisely when $G / K$ admits a wonderful completion (which always happens for $G / \bar{K}$ and $G / \mathrm{N}_{G}(K)$ ). Notice that, in general, the minimal spherical roots of $G / K$ do not necessarily belong to the root lattice, differently from the $\mathrm{n}$-spherical roots and from the sc-spherical roots which by construction are always in the root lattice of $G$.

Remark 4.1 The weight lattices of $G / K, G / \bar{K}$, and $G / \mathrm{N}_{G}(K)$ allow easily to compare $K, \bar{K}$, and $\mathrm{N}_{G}(K)$ : indeed, the quotient $\mathrm{N}_{G}(K) / K$ is diagonalizable (see [8, Corollaire 5.2]), and we have inclusions

$$
\Lambda_{G / \mathrm{N}_{G}(K)} \subset \Lambda_{G / \bar{K}} \subset \Lambda_{G / K},
$$


inducing isomorphisms (see, e.g., [12, Lemma 2.4])

$$
\begin{gathered}
x\left(\mathrm{~N}_{G}(K) / K\right) \simeq \Lambda_{G / K} / \Lambda_{G / \mathrm{N}_{G}(K),} \\
x\left(\mathrm{~N}_{G}(K) / \bar{K}\right) \simeq \Lambda_{G / \bar{K}} / \Lambda_{G / \mathrm{N}_{G}(K)}, \quad x(\bar{K} / K) \simeq \Lambda_{G / K} / \Lambda_{G / \bar{K}} .
\end{gathered}
$$

As already anticipated in the case of a symmetric variety, we define a fourth normalization for the spherical roots, setting

$$
\Delta_{G / K}=\left(\Delta_{G / K}^{\mathrm{n}} \backslash \Delta_{G / K}^{\dagger}\right) \cup \frac{1}{2} \Delta_{G / K}^{\dagger},
$$

where

$$
\Delta_{G / K}^{\dagger}=\left\{\sigma \in \Delta_{G / K}^{\mathrm{n}} \cap 2 \Delta \mid\left\langle\sigma, \tau^{\vee}\right\rangle \in 2 \mathbb{Z} \forall \tau \in \Delta_{G / K}^{\mathrm{n}}\right\} .
$$

Because $\Delta_{G / K}^{\mathrm{n}}$ is obtained from $\Delta_{G / K}^{\mathrm{sc}}$ by doubling some particular elements in $\Delta_{G / K}^{\mathrm{sc}} \cap \Delta$ (see [5, Section 2.4]), it turns out that we also have

$$
\Delta_{G / K}=\left(\Delta_{G / K}^{\mathrm{sc}} \backslash \Delta_{G / K}^{\dagger}\right) \cup \frac{1}{2} \Delta_{G / K}^{\dagger} .
$$

We denote by $\Phi_{G / K}$ the (reduced) root system generated by $\Delta_{G / K}$. Notice that $\Phi_{G / K}$ has also Weyl group $W_{G / K}$. In all cases that we will be interested (see Table 3), we have indeed $\Delta_{G / K}=\Delta_{G / K}^{\text {sc }}$ : this is always true when $K$ is connected.

If $\sigma \in \Delta_{G / K}^{\mathrm{n}}$, then the following three possibilities occur (see [7, Théorème 2.6]): either $\sigma \in \Phi^{+}$is a positive root, or $\sigma \in 2 \Delta$ is the double of a simple root, or $\sigma=\alpha+\beta$ is the sum of two strongly orthogonal positive roots (that is, neither $\alpha+\beta$ nor $\alpha-\beta$ is in $\Phi)$. Because $\Delta_{G / K}$ and $\Delta_{G / K}^{\text {sc }}$ are obtained from $\Delta_{G / K}^{\mathrm{n}}$ by replacing some elements in $2 \Delta \cap \Delta_{G / K}^{\mathrm{n}}$ with the corresponding simple roots, the same description applies to $\Delta_{G / K}^{\mathrm{sc}}$ and $\Delta_{G / K}$.

There is actually a finite list of possible cases: the shape of an n-spherical root, written as sum of simple roots, is necessarily one of those reported in Table 2 (where we also specify the type of the root subsystem of $\Phi$ generated by the support of the spherical root).

In general, $\Delta_{G / K}$ is not contained in $\Lambda_{G / K}$. Thus, we extend accordingly the weight lattice, and define $\Xi_{G / K}$ to be the lattice generated by $\Lambda_{G / K}$ together with $\Delta_{G / K}$, namely by $\Lambda_{G / K}$ and $\frac{1}{2} \Delta_{G / K}^{\dagger}$.

The following lemma will allow us to define the spherical coroots in $\Xi_{G / K}^{\vee}$, in analogy to what we did in the symmetric case.

Lemma 4.2 Let $G / K$ be a spherical variety, and let $\sigma \in \Delta_{G / K}^{\mathrm{sc}} \backslash \Phi^{+}$.

(i) If $\sigma=2 \alpha$ with $\alpha \in \Delta$, then $\left\langle\lambda, \alpha^{\vee}\right\rangle \in 2 \mathbb{Z}$ for all $\lambda \in \Xi_{G / K}$.

(ii) If $\sigma$ decomposes as a sum of two strongly orthogonal roots $\beta, \gamma \in \Phi^{+}$, then

$$
\beta_{\mid \Xi_{G / K}}^{\vee}=\gamma_{\mid \Xi_{G / K}}^{\vee}
$$

Moreover, any such decomposition of $\sigma$ yields the same result.

Proof (i) It is well known that $\langle\lambda, \alpha\rangle \in 2 \mathbb{Z}$ whenever $2 \alpha \in \Delta_{G / K}^{\text {sc }}$ (see [26, Section 1.4]). It is indeed being part of Luna's axioms which classify spherical varieties [5, Section 1.2]. Thus, by the definition of $\Delta_{G / K}^{\dagger}$, this property holds for all $\lambda \in \Xi_{G / K}$. 


\begin{tabular}{ll}
\hline$\sigma$ & Type of $\sigma$ \\
\hline$\alpha$ & $\mathrm{A}_{1}$ \\
$2 \alpha$ & $\mathrm{A}_{1}$ \\
$\alpha+\alpha^{\prime}$ & $\mathrm{A}_{1} \times \mathrm{A}_{1}$ \\
$\alpha_{1}+\cdots+\alpha_{m}$ & $\mathrm{~A}_{m}, m \geqslant 2$ \\
$\alpha_{1}+2 \alpha_{2}+\alpha_{3}$ & $\mathrm{~A}_{3}$ \\
$\alpha_{1}+\cdots+\alpha_{m}$ & $\mathrm{~B}_{m}, m \geqslant 2$ \\
$2\left(\alpha_{1}+\cdots+\alpha_{m}\right)$ & $\mathrm{B}_{m}, m \geqslant 2$ \\
$\alpha_{1}+2 \alpha_{2}+3 \alpha_{3}$ & $\mathrm{~B}_{3}$ \\
$\alpha_{1}+2\left(\alpha_{2}+\cdots+\alpha_{m-1}\right)+\alpha_{m}$ & $\mathrm{C}_{m}, m \geqslant 3$ \\
$2\left(\alpha_{1}+\cdots+\alpha_{m-2}\right)+\alpha_{m-1}+\alpha_{m}$ & $\mathrm{D}_{m}, m \geqslant 4$ \\
$\alpha_{1}+2 \alpha_{2}+3 \alpha_{3}+2 \alpha_{4}$ & $\mathrm{~F}_{4}$ \\
$\alpha_{1}+\alpha_{2}$ & $\mathrm{G}_{2}$ \\
$4 \alpha_{1}+2 \alpha_{2}$ & $\mathrm{G}_{2}$ \\
\hline
\end{tabular}

Table 2: sc-spherical roots

(ii) We point out that, in [21, Lemmas 6.2 and 6.4], a particular decomposition of $\sigma$ as a sum of two strongly orthogonal positive roots is considered, and the statement is proved for that decomposition.

Because $\Lambda_{G / K}^{+}=\Lambda_{G / \bar{K}}^{+}$and $\Delta_{G / K}^{\text {sc }}=\Delta_{G / \bar{K}}^{\text {sc }}$, we may assume that $K=\bar{K}$.

Scrolling through the list of Table 2, we see that the sc-spherical roots that are neither positive roots nor the double of a simple root are those of the following table (we also report an extra datum, which is defined here below).

\begin{tabular}{ccc}
\hline$\sigma$ & Type of $\sigma$ & $\operatorname{supp}(\sigma) \backslash \Delta^{p p}(\sigma)$ \\
\hline$\alpha+\alpha^{\prime}$ & $\mathrm{A}_{1} \times \mathrm{A}_{1}$ & $\left\{\alpha, \alpha^{\prime}\right\}$ \\
$\alpha_{1}+2 \alpha_{2}+\alpha_{3}$ & $\mathrm{~A}_{3}$ & $\left\{\alpha_{2}\right\}$ \\
$2\left(\alpha_{1}+\cdots+\alpha_{m}\right)$ & $\mathrm{B}_{m}, m \geqslant 2$ & $\left\{\alpha_{1}\right\}$ \\
$\alpha_{1}+2 \alpha_{2}+3 \alpha_{3}$ & $\mathrm{~B}_{3}$ & $\left\{\alpha_{3}\right\}$ \\
$2\left(\alpha_{1}+\cdots+\alpha_{m-2}\right)+\alpha_{m-1}+\alpha_{m}$ & $\mathrm{D}_{m}, m \geqslant 4$ & $\left\{\alpha_{1}\right\}$ \\
$4 \alpha_{1}+2 \alpha_{2}$ & $\mathrm{G}_{2}$ & $\left\{\alpha_{1}\right\}$ \\
\hline
\end{tabular}


The first case, with $\sigma$ of type $\mathrm{A}_{1} \times \mathrm{A}_{1}$, is somewhat special, and the statement is well known (see [26, Proposition 3.2]). It is indeed part of Luna's axioms to classify spherical varieties. Therefore, we will assume that $\operatorname{supp}(\sigma)$ is connected.

Denote

$$
\Delta_{G / K}^{p}=\left\{\alpha \in \Delta:\left\langle\lambda, \alpha^{\vee}\right\rangle=0 \forall \lambda \in \Omega_{G / K}^{+}\right\},
$$

and let $\Delta^{p p}(\sigma) \subset \operatorname{supp}(\sigma)$ be the set of simple roots orthogonal to $\sigma$. Then, we have $\Delta^{p p}(\sigma) \subset \Delta_{G / K}^{p}$ whenever $\sigma \notin \Phi^{+}$(see [5, Section 1.1]).

Notice that in all cases of the previous table with $\operatorname{supp}(\sigma)$ connected, there exists a unique simple root $\alpha \in \operatorname{supp}(\sigma)$ not orthogonal to $\sigma$. Therefore, it is enough to check that, for any spherical root $\sigma \in \Delta_{G / K}^{\text {sc }} \backslash\left(\Phi^{+} \cup 2 \Delta\right)$ and for any decomposition $\sigma=\beta+\gamma$ as a sum of two strongly orthogonal positive roots, it holds

$$
\left\langle\omega_{\alpha}, \beta^{\vee}\right\rangle=\left\langle\omega_{\alpha}, \gamma^{\vee}\right\rangle=1,
$$

which can easily be checked case by case.

For $\sigma \in \Delta_{G / K}$, using Lemma 4.2, we define the coroot $\sigma^{\vee} \in \Xi_{G / K}^{\vee}$ as follows:

(i) if $\sigma=2 \alpha$ with $\alpha \in \Delta$, then

$$
\sigma^{\vee}=\frac{1}{2} \alpha^{\vee}{ }_{\mid \Xi_{G / K}} \cdot
$$

(ii) if $\sigma=\beta+\gamma$ with $\beta, \gamma \in \Phi^{+}$strongly orthogonal, then

$$
\sigma^{\vee}=\beta_{\mid \Xi_{G / K}} \cdot
$$

(iii) if $\sigma=\beta$ with $\beta \in \Phi^{+}$, then

$$
\sigma^{\vee}=\beta^{\vee} \Xi_{G / K} .
$$

Set $\Delta_{G / K}^{\vee}=\left\{\sigma^{\vee} \mid \sigma \in \Delta_{G / K}\right\}$, then by construction we have the following.

Proposition 4.3 The triple $\mathcal{R}_{G / K}=\left(\Xi_{G / K}, \Delta_{G / K}, \Delta_{G / K}^{\vee}\right)$ is a based root datum. Moreover, the intersection $\Xi_{G / K} \cap \Lambda^{+}$is contained in the monoid of dominant weights of $\mathcal{R}_{G / K}$

$$
\Xi_{G / K}^{+}:=\left\{\lambda \in \Xi_{G / K}:\left\langle\lambda, \sigma^{\vee}\right\rangle \geqslant 0 \forall \sigma \in \Delta_{G / K}\right\} .
$$

Differently from the symmetric case, notice that the based root datum $\mathcal{R}_{G / K}$ is not necessarily semisimple: by Remark 4.1, this happens if and only if $K$ has finite index in its normalizer. When $\mathcal{R}_{G / K}$ is semisimple, every spherical weight is uniquely determined by its values against the spherical roots; otherwise, the extra information is obtained by restricting to the normalizer.

Lemma 4.4 Every $\lambda \in \Lambda_{G / K}$ is uniquely determined by its values against the spherical roots, together with its restriction to $B \cap \mathrm{N}_{G}(K)^{\circ}$.

Proof Denote $K^{\prime}=\mathrm{N}_{G}(K)^{\circ}$, then the restriction of characters to $B \cap K^{\prime}$ induces an exact sequence

$$
0 \longrightarrow \Lambda_{G / K^{\prime}} \longrightarrow \Lambda_{G / K} \longrightarrow X\left(B \cap K^{\prime}\right)^{B \cap K} \longrightarrow 0
$$


(see, e.g., [12, Lemma 2.4]). On the other hand, $\Lambda_{G / \bar{K}} \subset \Lambda_{G / K^{\prime}}$ is a sublattice of finite index, and $\Lambda_{G / \bar{K}}=\mathbb{Z} \Delta_{G / K}^{\mathrm{sc}}$; thus, every element in $\Lambda_{G / K^{\prime}}$ is uniquely determined by its values against the spherical roots.

\section{Affine spherical varieties with root system of type A}

We now consider generalizations of Conjecture 3.2 to other affine spherical homogeneous varieties for a simple algebraic group.

Let $(G, K)$ be a reductive spherical pair, and set $X=G / K$. Let $G_{X}$ be the connected reductive group defined by the root datum $\mathcal{R}_{X}=\left(\Xi_{X}, \Delta_{X}, \Delta_{X}^{\vee}\right)$, and correspondingly, let $T_{X} \subset G_{X}$ and $B_{X} \subset G_{X}$ be a maximal torus and a Borel subgroup containing $T_{X}$ inducing $\mathcal{R}_{X}$. For a spherical weight $\pi \in \Xi_{X}^{+}$(in particular, for $\pi \in \Lambda_{X}^{+}$), let $V_{X}(\pi)$ be the irreducible $G_{X}$-module with highest weight $\pi$. In analogy with the symmetric case, we denote by $\leqslant_{X}$ the partial order on $\Xi_{X}$ defined by $\Delta_{X}^{\mathrm{n}}$, namely

$$
\pi_{1} \leqslant X \pi_{2} \stackrel{\text { def }}{\Longleftrightarrow} \pi_{2}-\pi_{1} \in \mathbb{N} \Delta_{X}^{\mathrm{n}} .
$$

Conjecture 5.1 Let $(G, K)$ be a reductive spherical pair with $G$ simple and with $\Phi_{G / K}$ of type $A$, and set $X=G / K$. Let $\lambda, \mu, v \in \Lambda_{X}^{+}$, then

$$
E_{X}(v) \subset E_{X}(\lambda) \cdot E_{X}(\mu) \Longleftrightarrow\left\{\begin{array}{c}
V_{X}(v) \subset V_{X}(\lambda) \otimes V_{X}(\mu), \\
v \leqslant{ }_{X} \lambda+\mu .
\end{array}\right.
$$

With one exception (case Sph.A15 of Table 3), we will show that the previous conjecture holds true whenever $\Phi_{X}$ is a direct sum of rank 1 root systems-which happens in most of the cases-as a consequence of Stanley's Pieri rule for Jack symmetric functions [33, Theorem 6.1]. More generally, we will show that in all cases but case Sph.A15, the previous conjecture follows from Conjecture 3.2 (and in particular from Stanley's Conjecture 3.1). Finally, in the remaining case Sph.A15, the conjecture is supported by computational experiments.

For the remainder of the section, $G$ will be a simple and simply connected algebraic group, and $K \subset G$ a connected reductive spherical subgroup such that the spherical root system $\Phi_{G / K}$ is of type A. A list of such spherical pairs is given in Table 3 (where we omit the cases of Table 1$)$. For a full list of the reductive spherical pairs $(G, K)$ with $G$ simple and $K$ connected, we refer to $[19,23]$.

Remark 5.2 As already pointed out in Remark 3.4 for the symmetric case, also in this case, it is possible to formulate Conjecture 5.1 using the based root datum $\mathcal{R}_{X}^{\mathrm{n}}=$ $\left(\Lambda_{X}, \Delta_{X}^{\mathrm{n}}, \Delta_{X}^{\mathrm{n} \vee}\right)$ rather than $\mathcal{R}_{X}$, and defining the reductive group $G_{X}$ accordingly. Using $\mathcal{R}_{X}^{\mathrm{n}}$, the formulation becomes also simpler, because the inequality $v \leqslant_{X} \lambda+\mu$ becomes redundant. However, the formulation that we adopted seems to be preferable, as it fits inside the more general framework that we will consider in Section 6. Notice also that in all cases but Sph.A10, it holds $\Delta_{X}=\Delta_{X}^{\mathrm{n}}$, so that, in these cases, we have indeed $\mathcal{R}_{X}=\mathcal{R}_{X}^{\mathrm{n}}$.

Remark 5.3 Notice that the cases Sph.A7, Sph.A11, and Sph.A15 are actually symmetric, with nonreduced restricted root system of type $B C_{1}$. 


\begin{tabular}{lccc}
\hline & $G$ & $K$ & $\Phi_{G / K}$ \\
\hline Sph.A6 & $\mathrm{SL}(n), n \geqslant 3$ & $\mathrm{SL}(n-1)$ & $\mathrm{A}_{1}$ \\
Sph.A7 & $\mathrm{SL}(n), n \geqslant 3$ & $\mathrm{GL}(n-1)$ & $\mathrm{A}_{1}$ \\
Sph.A8 & $\mathrm{SL}(2 n+1), n \geqslant 2$ & $\mathrm{Sp}(2 n)$ & $\mathrm{A}_{n} \times \mathrm{A}_{n-1}$ \\
Sph.A9 & $\mathrm{SL}(2 n+1), n \geqslant 2$ & $\mathrm{GL}(1) \times \mathrm{Sp}(2 n)$ & $\mathrm{A}_{n} \times \mathrm{A}_{n-1}$ \\
Sph.A10 & $\mathrm{Sp}(2 n), n \geqslant 2$ & $\mathrm{GL}(1) \times \mathrm{Sp}(2 n-2)$ & $\mathrm{A}_{1} \times \mathrm{A}_{1}$ \\
Sph.A11 & $\mathrm{Sp}(2 n), n \geqslant 3$ & $\mathrm{Sp}(2) \times \mathrm{Sp}(2 n-2)$ & $\mathrm{A}_{1}$ \\
Sph.A12 & $\mathrm{Spin}(7)$ & $\mathrm{G} 2$ & $\mathrm{~A}_{1}$ \\
Sph.A13 & $\mathrm{Spin}(9)$ & $\mathrm{Spin}(7)$ & $\mathrm{A}_{1} \times \mathrm{A}_{1}$ \\
Sph.A14 & $\mathrm{Spin}(8)$ & $\mathrm{G}$ & $\mathrm{A}_{1} \times \mathrm{A}_{1} \times \mathrm{A}_{1}$ \\
Sph.A15 & $\mathrm{F}_{4}$ & $\mathrm{Spin}(9)$ & $\mathrm{A}_{1}$ \\
Sph.A16 & $\mathrm{G}_{2}$ & $\mathrm{SL}(3)$ & $\mathrm{A}_{1}$ \\
\hline
\end{tabular}

Table 3: Reductive spherical pairs $(G, K)$ with root system of type A.

Notice also that the unique cases where $\Phi_{G / K}$ is not a direct sum of subsystems of type $A_{1}$ are cases Sph.A8 and Sph.A9. In the first case, $G / K$ is the principal model variety of $\operatorname{SL}(n)$ : a homogeneous variety $G / H$ is said to be a model variety for $G$ if it is spherical and $\Lambda_{G / K}^{+}=X(T)^{+}$(see $[13,14]$ ). In the latter case, $G / K$ is the homogeneous space which corresponds to the model variety of $\operatorname{SL}(n)$ in Luna's classification [27].

All other cases, where $\Phi_{G / K}$ is a direct sum of subsystems of type $A_{1}$, are related to some transitive action of a compact Lie group on a projective space, on a sphere, or on a product of spheres. Let indeed $G_{\mathbb{R}} \subset G$ and $K_{\mathbb{R}} \subset K$ be the associated compact real forms, then we have the following descriptions for $G_{\mathbb{R}} / K_{\mathbb{R}}$ :

(i) It is isomorphic to $\mathbb{P}^{n}(\mathbb{C})$ in case Sph.A7, to $\mathbb{P}^{2 n}(\mathbb{C})$ in case Sph.A10, to $\mathbb{P}^{n}(\mathbb{H})$ in case Sph.A11, and to $\mathbb{P}^{2}(\mathbb{O})$ in case Sph.A15 (where $\mathbb{H}$ and $\mathbb{O}$ denote, respectively, the algebras of the quaternions and of the octonions).

(ii) It is isomorphic to $\mathbb{S}^{2 n-1}$ in case Sph.A6, to $\mathbb{S}^{7}$ in case Sph.A12, to $\mathbb{S}^{15}$ in case Sph.A13, to $\mathbb{S}^{6}$ in case Sph.A16, and to $\mathbb{S}^{7} \times \mathbb{S}^{7}$ in case Sph.A14.

By a theorem of Cartan, if $G_{\mathbb{R}} / H_{\mathbb{R}}$ is an irreducible compact Riemannian symmetric space of rank 1 , then $H_{\mathbb{R}}$ acts transitively on the unit sphere in the isotropy representation of $H_{\mathbb{R}}$. This gives indeed rise to the transitive actions on spheres which correspond to cases Sph.A6 and Sph.A13 (which, respectively, come from the families of symmetric spaces corresponding to cases Sph.A7 and Sph.A15). More generally, transitive actions of compact Lie groups on spheres were classified by MontgomerySamelson and Borel: those which are not related to a symmetric space of rank 1 correspond to cases Sph.A12 and Sph.A13 (see, e.g., [32]). 
There is indeed another transitive action on a sphere which is associated to a rank 1 symmetric space, which is not included in Table 3: it is the transitive action of $\operatorname{Sp}(2, \mathbb{R}) \times \operatorname{Sp}(2 n, \mathbb{R})$ on $\mathbb{S}^{4 n-1}$ (which comes from the family of symmetric spaces corresponding to case Sph.A11). This action corresponds to the reductive spherical pair

$$
(G, K)=(\operatorname{Sp}(2) \times \operatorname{Sp}(2 n), \operatorname{diag}(\operatorname{Sp}(2)) \times \operatorname{Sp}(2 n-2)),
$$

in which case $\Phi_{G / K}$ is of type $A_{1} \times A_{1}$. Even though $G$ is not simple, this case also fits in the general framework which allows us to deal with the cases of Table 3 . We will analyze this case separately in Remark 5.11 .

To deal with the cases of Table 3, a first useful remark is that cases Sph.A7, Sph.A9, and Sph.A11, respectively, follow (almost immediately) from the companion cases Sph.A6, Sph.A8, and Sph.A10. This will be explained in Section 5.3.

All the remaining cases of Table 3-with the exception of case Sph.A15-will follow from some cases of Table 1, thanks to a related factorization of a simple algebraic group as a product of two reductive subgroups. Such factorizations have been classified by Onishchik [29] in the context of compact Lie groups (see also [15, Sections 4.5 and 4.6]) and by Liebeck, Saxl, and Seitz algebraically in arbitrary characteristic [24]. More precisely, in all the considered cases, the multiplication will reduce to that of some symmetric varieties, which correspond to a decomposition of $\Phi_{G / K}$ into two orthogonal components.

In particular, we will find a reductive overgroup $\widehat{G} \supset G$ and a symmetric subgroup $\widehat{K} \subset \widehat{G}$ such that $\widehat{G}=G \cdot \widehat{K}$ and $K=G \cap \widehat{K}$, yielding a $G$-equivariant isomorphism

$$
G / K \simeq \widehat{G} / \widehat{K}
$$

and inducing a natural projection of weight monoids

$$
\hat{\varphi}: \Lambda_{G / K}^{+} \longrightarrow \Lambda_{\widehat{G} / \widehat{K}}^{+} .
$$

As a consequence of a theorem of Luna [25], notice that in the previous setting $G / K$ and $\widehat{G} / \widehat{K}$ are isomorphic as $G$-varieties if and only if $G / K$ embeds $G$-equivariantly into $\widehat{G} / \widehat{K}$ as a dense open subset.

We will also need to bring into the picture a second symmetric pair $(H, K)$ (possibly trivial), where $H$ is a suitable connected reductive subgroup of $G$ containing $K$ and the connected center of $\mathrm{N}_{G}(K)$, yielding an $H$-equivariant embedding

$$
H / K \hookrightarrow G / K
$$

and inducing a second projection of weight monoids

$$
\bar{\varphi}: \Lambda_{G / K}^{+} \longrightarrow \Lambda_{H / K}^{+} \cdot
$$

The two maps $\hat{\varphi}$ and $\bar{\varphi}$ will allow us to identify the spherical functions of $G / K$, and describe their products, in terms of those of $\widehat{G} / \widehat{K}$ and $H / K$. Before treating the various cases in detail, let us explain here the general argument.

Suppose that we have:

(i) a connected reductive overgroup $\widehat{G} \supset G$ and a spherical subgroup $\widehat{K} \subset \widehat{G}$ such that $G / K \simeq \widehat{G} / \widehat{K}$, and 
(ii) a connected reductive subgroup $H \subset G$ containing $K$ (and the connected center of $\mathrm{N}_{G}(K)$ ).

We also assume (it will always be true in our cases) that there is a compatible choice of Borel subgroups, that is, there exists a maximal torus and Borel subgroup $\widehat{T} \subset \widehat{B}$ of $\widehat{G}$ with $\widehat{B} \widehat{K}$ open in $\widehat{G}$ such that:

(i) $T=\widehat{T} \cap G$ is a maximal torus of $G$, and $B=\widehat{B} \cap G$ is a Borel subgroup of $G$ with $B K$ is open in $G$.

(ii) $T_{H}=T \cap H$ is a maximal torus of $H$, and $B_{H}=B \cap H$ is a Borel subgroup of $H$ with $B_{H} K$ is open in $H$.

On the one hand, the restriction of the $\widehat{G}$-action to $G$ induces an isomorphism of $G$-modules

$$
\bigoplus_{\mu \in \Lambda_{\widehat{G} / \widehat{K}}^{+}} E_{\widehat{G} / \widehat{K}}(\mu)=\mathbb{C}[\widehat{G} / \widehat{K}] \stackrel{\sim}{\longrightarrow} \mathbb{C}[G / K]=\bigoplus_{\lambda \in \Lambda_{G / K}^{+}} E_{G / K}(\lambda) .
$$

Thus, for all $\lambda \in \Lambda_{G / K}^{+}$, there exists a unique $\hat{\lambda} \in \Lambda_{\widehat{G} / \widehat{K}}^{+}$such that

$$
\operatorname{Hom}_{G}\left(V_{\widehat{G}}(\hat{\lambda}), V_{G}(\lambda)\right) \neq 0,
$$

and we define $\hat{\varphi}(\lambda)=\hat{\lambda}$.

On the other hand, by definition, for all $\lambda \in \Lambda_{G / K}^{+}$, the invariant space $V_{G}(\lambda)^{K}$ is one-dimensional. Looking at the decomposition of $V_{G}(\lambda)$ into simple $H$-modules, it follows that there exists a unique $\bar{\lambda} \in \Lambda_{H / K}^{+}$such that

$$
\operatorname{Hom}_{H}\left(V_{G}(\lambda), V_{H}(\bar{\lambda})\right) \neq 0
$$

(and the dimension of the latter is necessarily 1). Thus, we define $\bar{\varphi}(\lambda)=\bar{\lambda}$.

Consider now the restriction of functions from $G / K$ to $H / K$

$$
\bigoplus_{\lambda \in \Lambda_{G / K}^{+}} E_{G / K}(\lambda)=\mathbb{C}[G / K] \stackrel{\rho}{\longrightarrow} \mathbb{C}[H / K]=\bigoplus_{\mu \in \Lambda_{H / K}^{+}} E_{H / K}(\mu) .
$$

In the following lemma, we show that $\rho\left(E_{G / K}(\lambda)\right)$ is nonzero; hence, it coincides with $E_{H / K}(\bar{\lambda})$.

Lemma 5.4 Let $V \subset \mathbb{C}[G / K]$ be an irreducible G-submodule. Then, $\rho(V) \subset \mathbb{C}[H / K]$ is an irreducible H-submodule.

Proof By the discussion above, we only need to show that $\rho(V) \neq 0$.

Suppose that $V \simeq V_{G}(\lambda)$. Fix $K$-invariant vectors $v_{\lambda} \in V_{G}(\lambda)$ and $\psi_{\lambda} \in V_{G}(\lambda)^{*}$, and define

$$
f_{G, \lambda}(g K)=\left\langle\psi_{\lambda}, g \cdot v_{\lambda}\right\rangle
$$

Then, $f_{G, \lambda} \in V^{K}$ is a nonzero $K$-invariant function.

Similarly, if $v_{\bar{\lambda}} \in V_{H}(\bar{\lambda})$ and $\psi_{\bar{\lambda}} \in V_{H}(\bar{\lambda})^{*}$ are $K$-invariant vectors, setting

$$
f_{H, \bar{\lambda}}(h K)=\left\langle\psi_{\bar{\lambda}}, h \cdot v_{\bar{\lambda}}\right\rangle,
$$

we get a nonzero $K$-invariant function on $H / K$. 
On the other hand, there is a unique $H$-equivariant embedding of $V_{H}(\bar{\lambda})$ inside $V_{G}(\lambda)$, and a unique $H$-equivariant embedding of $V_{H}(\bar{\lambda})^{*}$ inside $V_{G}(\lambda)^{*}$. With respect to such embeddings, we have equalities

$$
V_{G}(\lambda)^{K}=V_{H}(\bar{\lambda})^{K} \quad\left(V_{G}(\lambda)^{*}\right)^{K}=\left(V_{H}(\bar{\lambda})^{*}\right)^{K} .
$$

Because these invariant subspaces are one-dimensional, it follows that the $K$-invariant vectors $v_{\lambda}$ and $v_{\bar{\lambda}}$ are proportional, and similarly for the $K$-invariant linear functions $\psi_{\lambda}$ and $\psi_{\bar{\lambda}}$. Thus, the restriction $\rho\left(f_{G, \lambda}\right)$ is a nonzero scalar multiple of $f_{H, \bar{\lambda}}$.

We now show that the two maps $\hat{\varphi}$ and $\bar{\varphi}$, defined above, are actually morphisms of monoids.

Proposition 5.5 With notations and hypotheses as above, both the maps

$$
\hat{\varphi}: \Lambda_{G / K}^{+} \longrightarrow \Lambda_{\widehat{G} / \widehat{K}}^{+} \text {and } \bar{\varphi}: \Lambda_{G / K}^{+} \longrightarrow \Lambda_{H / K}^{+}
$$

are additive maps.

Proof (i) Let $\Gamma(\widehat{G}, G)$ be the branching monoid associated to the pair $(\widehat{G}, G)$, namely

$$
\Gamma(\widehat{G}, G)=\left\{(\pi, \lambda) \in X(\widehat{T})^{+} \times X(T)^{+} \mid \operatorname{Hom}_{G}\left(V_{G}(\lambda), V_{\widehat{G}}(\pi)\right) \neq 0\right\} .
$$

This is indeed a finitely generated monoid (see [1]).

Consider the projections

$$
p: \Gamma(\widehat{G}, G) \rightarrow X(\widehat{T})^{+}, \quad q: \Gamma(\widehat{G}, G) \rightarrow X(T)^{+} .
$$

Because $K$ is spherical in $G$, notice that $p^{-1}\left(\Lambda_{\widehat{G} / \widehat{K}}^{+}\right)$is a submonoid of $\Gamma(\widehat{G}, G)$, and that $q$ induces an isomorphism $p^{-1}\left(\Lambda_{\widehat{G} / \widehat{K}}^{+}\right) \rightarrow \Lambda_{G / K}^{+}$.

If indeed $(\pi, \lambda) \in \Gamma(\widehat{G}, G)$ and $\pi \in \Lambda_{\widehat{G} / \widehat{K}}^{+}$, then by the isomorphism $\mathbb{C}[G / K] \simeq$ $\mathbb{C}[\widehat{G} / \widehat{K}]$, it follows $\lambda \in \Lambda_{G / K}^{+}$. Conversely, if $\lambda \in \Lambda_{G / K}^{+}$, because $\mathbb{C}[G / K] \simeq \mathbb{C}[\widehat{G} / \widehat{K}]$, the multiplicity-free property implies that there is a unique $\pi \in \Lambda_{\widehat{G} / \widehat{K}}^{+}$with $(\pi, \lambda) \epsilon$ $\Gamma(\widehat{G}, G)$, and by definition $\pi=\hat{\varphi}(\lambda)$.

It is now clear that, under the isomorphism $\Lambda_{G / K}^{+} \simeq p^{-1}\left(\Lambda_{\widehat{G} / \widehat{K}}^{+}\right)$, the map $\hat{\varphi}$ coincides with the restriction of the projection $p$. In particular, we see that $\hat{\varphi}$ is an additive map.

(ii) The proof that $\bar{\varphi}$ is additive is similar to the previous one, by considering the branching monoid associated to the pair $(G, H)$

$$
\Gamma(G, H)=\left\{(\lambda, \pi) \in \mathcal{X}(T)^{+} \times \mathcal{X}\left(T_{H}\right)^{+} \mid \operatorname{Hom}_{H}\left(V_{H}(\pi), V_{G}(\lambda)\right) \neq 0\right\}
$$

together with the projections

$$
p: \Gamma(G, H) \rightarrow X(T)^{+}, \quad q: \Gamma(G, H) \rightarrow X\left(T_{H}\right)^{+} .
$$

Then, $p$ induces an isomorphism of monoids $q^{-1}\left(\Lambda_{H / K}^{+}\right) \rightarrow \Lambda_{G / K}^{+}$, and $\bar{\varphi}$ corresponds to the restriction of $q$.

Therefore, we can extend $\hat{\varphi}$ and $\bar{\varphi}$ to morphisms of the associated weight lattices. When the lattice $\Xi_{G / K}$ is bigger than $\Lambda_{G / K}$, we will see in the cases under consideration 
that the extensions of $\hat{\varphi}$ and $\bar{\varphi}$ to $\Xi_{G / K}$, respectively, take values in $\Xi_{\widehat{G} / \widehat{K}}$ and $\Xi_{H / K}$. The description of the maps $\hat{\varphi}$ and $\bar{\varphi}$ will immediately imply the following.

Proposition 5.6 Let $(G, K)$ be a reductive spherical pair with $\Phi_{G / K}$ of type $A$, and suppose that we are in one of the following cases of Table 3:

Sph.A6, Sph.A8, Sph.A10, Sph.A12, Sph.A13, Sph.A14, and Sph.A16.

Then, there exist a connected reductive subgroup $H \subset G$ containing $K$ (possibly equal to $K$ ), a connected reductive overgroup $\widehat{G} \supset G$, and a symmetric subgroup $\widehat{K} \subset \widehat{G}$ with $\widehat{K} \cap G=K$ such that the map

$$
(\hat{\varphi}, \bar{\varphi}): \Xi_{G / K} \longrightarrow \Xi_{\widehat{G} / \widehat{K}} \oplus \Xi_{H / K}
$$

is an isogeny of based root data

$$
\mathcal{R}_{G / K} \longrightarrow \mathcal{R}_{\widehat{G} / \widehat{K}} \oplus \mathcal{R}_{H / K}
$$

To be clear about notation and terminology, $\mathcal{R}_{\widehat{G} / \widehat{K}} \oplus \mathcal{R}_{H / K}$ is the based root datum defined by

$$
\left(\Xi_{\widehat{G} / \widehat{K}} \oplus \Xi_{H / K}, \Delta_{\widehat{G} / \widehat{T}} \sqcup \Delta_{H / K}, \Delta_{\widehat{G} / \widehat{T}}^{\vee} \sqcup \Delta_{H / K}^{\vee}\right),
$$

and $\varphi=(\hat{\varphi}, \bar{\varphi})$ is an isogeny of based root data in the following (usual) sense:

(i) $\varphi: \Xi_{G / K} \rightarrow \Xi_{\widehat{G} / \widehat{K}} \oplus \Xi_{H / K}$ is injective with finite cokernel.

(ii) $\varphi$ restricts to a bijection between $\Delta_{G / K}$ and $\Delta_{\widehat{G} / \widehat{T}} \sqcup \Delta_{H / K}$.

(iii) The dual map satisfies $\varphi^{\vee}\left(\varphi(\sigma)^{\vee}\right)=\sigma^{\vee}$, for all $\sigma \in \Delta_{G / K}$.

In particular, we stress the following property.

Corollary 5.7 Every weight in $\Lambda_{G / K}$ is uniquely determined by its images in $\Lambda_{\widehat{G} / \widehat{K}}$ and in $\Lambda_{H / K}$.

In the next two subsections, we will deal with the various cases of Proposition 5.6.

For every spherical pair $(G, K)$, we provide the groups $\widehat{G}, \widehat{K}$, and $H$ with the required properties. The inclusions among the given groups are well known, and all the mentioned properties can be read off from the classification of the factorizations of simple algebraic groups $[24,29]$ (see also [15, Sections 4.5 and 4.6]).

For each affine spherical variety $G / K, \widehat{G} / \widehat{K}$, and $H / K$, we describe the sets of spherical roots $\Delta_{G / K}, \Delta_{\widehat{G} / \widehat{K}}$, and $\Delta_{H / K}$ (which we call normalized spherical roots), and minimal set of generators of the monoids $\Lambda_{G / K}^{+}, \Lambda_{\widehat{G} / \widehat{K}}^{+}$, and $\Lambda_{H / K}^{+}$(which we call fundamental spherical weights). These data are also well known, and can be read off, for example, from [6].

Finally, in each case, we describe the maps $\hat{\varphi}$ and $\bar{\varphi}$. Being additive, it is enough to describe the images of the fundamental spherical weights of $G / K$.

\subsection{Indecomposable cases}

We consider here the cases Sph.A12 and Sph.A16 where we take the subgroup $H \subset G$ as $K$ itself (thus, $\Xi_{H / K}=0$ and $\Delta_{H / K}=\varnothing$ ). The overgroup $\widehat{G} \supset G$ and the symmetric subgroup $\widehat{K} \subset \widehat{G}$ with the prescribed properties are given below. 
From its explicit description, it will be clear that the map

$$
\hat{\varphi}: \Lambda_{G / K} \longrightarrow \Lambda_{\widehat{G} / \widehat{K}}
$$

induces an isomorphism of root data

$$
\mathcal{R}_{G / K} \stackrel{\sim}{\longrightarrow} \mathcal{R}_{\widehat{G} / \widehat{K}} .
$$

(Sph.A12) $G / K=\operatorname{Spin}(7) / G_{2}$

$$
\widehat{G} / \widehat{K}=\operatorname{SO}(8) / \operatorname{SO}(7)
$$

\begin{tabular}{|c|c|c|}
\hline & Norm. spherical roots & Fund. sph. weights \\
\hline \hline$G / K$ & $\alpha_{1}+2 \alpha_{2}+3 \alpha_{3}$ & $\omega_{3}$ \\
\hline$\widehat{G} / \widehat{K}$ & $2\left(\alpha_{1}^{\prime}+\alpha_{2}^{\prime}\right)+\alpha_{3}^{\prime}+\alpha_{4}^{\prime}$ & $\omega_{1}^{\prime}$ \\
\hline
\end{tabular}

$$
\hat{\varphi}: a \omega_{3} \longmapsto a \omega_{1}^{\prime}
$$

(Sph.A16) $G / K=\mathrm{G}_{2} / \mathrm{SL}(3)$

$$
\widehat{G} / \widehat{K}=\operatorname{SO}(7) / \operatorname{SO}(6)
$$

\begin{tabular}{|c|c|c|}
\hline & Norm. spherical roots & sph. weights \\
\hline \hline$G / K$ & $4 \alpha_{1}+2 \alpha_{2}$ & $\omega_{1}$ \\
\hline$\widehat{G} / \widehat{K}$ & $2\left(\alpha_{1}^{\prime}+\alpha_{2}^{\prime}+\alpha_{3}^{\prime}\right)$ & $\omega_{1}^{\prime}$ \\
\hline
\end{tabular}

$$
\hat{\varphi} \quad: \quad a \omega_{1} \longmapsto a \omega_{1}^{\prime}
$$

Proposition 5.8 Conjecture 5.1 holds true if $(G, K)$ is one of the spherical pairs Sph.A12 and Sph.A16 of Table 3.

Proof By restricting the action from $\widehat{G}$ to $G$, every spherical module for the pair $(\widehat{G}, \widehat{K})$ remains irreducible and yields a spherical module for the pair $(G, K)$. This gives a canonical bijection between the two: the restriction of every irreducible $\widehat{G}$ module containing a nontrivial $\widehat{K}$-invariant subspace is irreducible as a $G$-module as well, and it is obviously $K$-spherical. 
In particular, the decomposition of the product of two spherical functions of $G / K$ immediately reduces to the decomposition of the product of the corresponding spherical functions of $\widehat{G} / \widehat{K}$.

By Stanley's Pieri rule for Jack symmetric functions, we know that Conjecture 3.2 holds true for the symmetric pair $(\widehat{G}, \widehat{K})$. On the other hand, $\hat{\varphi}$ induces an isomorphism of root data $\mathcal{R}_{G / K} \simeq \mathcal{R}_{\widehat{G} / \widehat{K}}$. Thus, Conjecture 5.1 holds true for $(G, K)$ as well.

\subsection{Decomposable cases}

We now consider the cases Sph.A6, Sph.A8, Sph.A10, Sph.A13, and Sph.A14 of Table 3: we define the subgroup $H \subset G$, the overgroup $\widehat{G} \supset G$, and the symmetric subgroup $\widehat{K} \subset \widehat{G}$ with the required properties. Notice that $H$ will always contain the connected center of $\mathrm{N}_{G}(K)$. As already mentioned, for the given inclusions among groups and their related properties, one can see $[24,29]$ and also [15, Sections 4.5 and 4.6].

We also provide an explicit description of the maps

$$
\hat{\varphi}: \Lambda_{G / K} \longrightarrow \Lambda_{\widehat{G} / \widehat{K}} \text { and } \bar{\varphi}: \Lambda_{G / K} \longrightarrow \Lambda_{H / K} .
$$

Proposition 5.6 will be an immediate consequence of these descriptions.

(Sph.A6) $G / K=\operatorname{SL}(n) / \operatorname{SL}(n-1)$

$$
\begin{aligned}
\widehat{G} / \widehat{K} & =\operatorname{SO}(2 n) / \operatorname{SO}(2 n-1), \\
H / K & =\operatorname{GL}(n-1) / \operatorname{SL}(n-1) .
\end{aligned}
$$

\begin{tabular}{|c|c|c|}
\hline & Normalized spherical roots & Fund. sph. weights \\
\hline \hline$G / K$ & $\alpha_{1}+\cdots+\alpha_{n-1}$ & $\omega_{1}, \omega_{n-1}$ \\
\hline$\widehat{G} / \widehat{K}$ & $2\left(\alpha_{1}^{\prime}+\cdots+\alpha_{n-2}^{\prime}\right)+\alpha_{n-1}^{\prime}+\alpha_{n}^{\prime}$ & $\omega_{1}^{\prime}$ \\
\hline$H / K$ & none & $\pm \omega_{n-1}^{\prime \prime}$ \\
\hline
\end{tabular}

$$
\begin{array}{|lllll|}
\hline \hat{\varphi} \quad: \quad a \omega_{1}+b \omega_{n-1} & \longmapsto & (a+b) \omega_{1}^{\prime} \\
\hline \bar{\varphi} \quad: & a \omega_{1}+b \omega_{n-1} & \longmapsto & (b-a) \omega_{n-1}^{\prime \prime} \\
\hline
\end{array}
$$

(Sph.A8) $G / K=\operatorname{SL}(2 n+1) / \operatorname{Sp}(2 n)$

$$
\begin{gathered}
\widehat{G} / \widehat{K}=\operatorname{SL}(2 n+2) / \operatorname{Sp}(2 n+2), \\
H / K=\operatorname{GL}(2 n) / \operatorname{Sp}(2 n) .
\end{gathered}
$$

\begin{tabular}{|c|c|c|}
\hline & Normalized spherical roots & Fund. spherical weights \\
\hline \hline$G / K$ & $\alpha_{i}+\alpha_{i+1}(i=1, \ldots, 2 n-1)$ & $\omega_{i}(i=1, \ldots, 2 n)$ \\
\hline$\widehat{G} / \widehat{K}$ & $\alpha_{2 i-1}^{\prime}+2 \alpha_{2 i}^{\prime}+\alpha_{2 i+1}^{\prime}(i=1, \ldots, n)$ & $\omega_{2 i}^{\prime}(i=1, \ldots, n)$ \\
\hline$H / K$ & $\alpha_{2 i-1}^{\prime \prime}+2 \alpha_{2 i}^{\prime \prime}+\alpha_{2 i+1}^{\prime \prime}(i=1, \ldots, n-1)$ & $\omega_{2 i}^{\prime \prime}(i=1, \ldots, n-1), \pm \omega_{2 n}^{\prime \prime}$ \\
\hline
\end{tabular}




\begin{tabular}{|lllll|}
\hline$\hat{\varphi}$ & $:$ & $\sum_{i=1}^{2 n} a_{i} \omega_{i}$ & $\longmapsto$ & $\sum_{i=1}^{n}\left(a_{2 i-1}+a_{2 i}\right) \omega_{2 i}^{\prime}$ \\
\hline $\bar{\varphi}$ & $:$ & $\sum_{i=1}^{2 n} a_{i} \omega_{i}$ & $\longmapsto$ & $\sum_{i=1}^{n-1}\left(a_{2 i}+a_{2 i+1}\right) \omega_{2 i}^{\prime \prime}+\left(a_{2 n}-\sum_{i=1}^{n} a_{2 i-1}\right) \omega_{2 n}^{\prime \prime}$ \\
\hline
\end{tabular}

(Sph.A10) $G / K=\operatorname{Sp}(2 n) /[\mathrm{GL}(1) \times \operatorname{Sp}(2 n-2)]$

$$
\begin{gathered}
\widehat{G} / \widehat{K}=\operatorname{SL}(2 n) / \operatorname{GL}(2 n-1), \\
H / K=\operatorname{Sp}(2) / \operatorname{GL}(1) .
\end{gathered}
$$

\begin{tabular}{|c|c|c|}
\hline & Normalized spherical roots & Fund. sph. weights \\
\hline \hline$G / K$ & $\alpha_{1}, \alpha_{1}+2\left(\alpha_{2}+\cdots+\alpha_{n-1}\right)+\alpha_{n}$ & $2 \omega_{1}, \omega_{2}$ \\
\hline$\widehat{G} / \widehat{K}$ & $\alpha_{1}^{\prime}+\cdots+\alpha_{2 n-1}^{\prime}$ & $\omega_{1}^{\prime}+\omega_{2 n-1}^{\prime}$ \\
\hline$H / K$ & $\alpha_{1}^{\prime \prime}$ & $2 \omega_{1}^{\prime \prime}$ \\
\hline
\end{tabular}

\begin{tabular}{|lllll|}
\hline$\hat{\varphi}:$ & $2 a \omega_{1}+b \omega_{2}$ & $\longmapsto$ & $(a+b)\left(\omega_{1}^{\prime}+\omega_{2 n-1}^{\prime}\right)$ \\
\hline $\bar{\varphi}:$ & $2 a \omega_{1}+b \omega_{2}$ & $\longmapsto$ & $2 a \omega_{1}^{\prime \prime}$ \\
\hline
\end{tabular}

(Sph.A13) $G / K=\operatorname{Spin}(9) / \operatorname{Spin}(7)$

$$
\begin{aligned}
\widehat{G} / \widehat{K} & =\operatorname{SO}(16) / \operatorname{SO}(15), \\
H / K & =\operatorname{Spin}(8) / \operatorname{Spin}(7) .
\end{aligned}
$$

\begin{tabular}{|c|c|c|}
\hline & Normalized spherical roots & Fund. sph. weights \\
\hline \hline$G / K$ & $\alpha_{1}+\alpha_{2}+\alpha_{3}+\alpha_{4}, \alpha_{2}+2 \alpha_{3}+3 \alpha_{4}$ & $\omega_{1}, \omega_{4}$ \\
\hline$\widehat{G} / \widehat{K}$ & $2\left(\alpha_{1}^{\prime}+\cdots+\alpha_{6}^{\prime}\right)+\alpha_{7}^{\prime}+\alpha_{8}^{\prime}$ & $\omega_{1}^{\prime}$ \\
\hline$H / K$ & $\alpha_{1}^{\prime \prime}+2 \alpha_{2}^{\prime \prime}+\alpha_{3}^{\prime \prime}+2 \alpha_{4}^{\prime \prime}$ & $\omega_{4}^{\prime \prime}$ \\
\hline
\end{tabular}

$$
\begin{array}{|llll|}
\hline \hat{\varphi} \quad: \quad a \omega_{1}+b \omega_{4} & \longrightarrow & (2 a+b) \omega_{1}^{\prime} \\
\hline \bar{\varphi}: & a \omega_{1}+b \omega_{4} & \longmapsto & b \omega_{4}^{\prime \prime} \\
\hline
\end{array}
$$

(Sph.A14) $G / K=\operatorname{Spin}(8) / G_{2}$

$$
\widehat{G} / \widehat{K}=\operatorname{Spin}(8) / \operatorname{Spin}(7) \times \operatorname{Spin}(8) / \operatorname{Spin}(7)
$$

(where the two copies of $\operatorname{Spin}(7)$ are not conjugated in $\operatorname{Spin}(8)$ ),

$$
H / K=\operatorname{Spin}(7) / G_{2} \text {. }
$$




\begin{tabular}{|c|c|c|}
\hline & Normalized spherical roots & Fund. sph. weights \\
\hline \hline$G / K$ & $\alpha_{1}+\alpha_{2}+\alpha_{3}, \alpha_{1}+\alpha_{2}+\alpha_{4}, \alpha_{2}+\alpha_{3}+\alpha_{4}$ & $\omega_{1}, \omega_{3}, \omega_{4}$ \\
\hline$\widehat{G} / \widehat{K}$ & $2 \alpha_{1}^{\prime}+2 \alpha_{2}^{\prime}+\alpha_{3}^{\prime}+\alpha_{4}^{\prime}, \alpha_{1}^{\prime \prime}+2 \alpha_{2}^{\prime \prime}+2 \alpha_{3}^{\prime \prime}+\alpha_{4}^{\prime \prime}$ & $\omega_{1}^{\prime}, \omega_{3}^{\prime \prime}$ \\
\hline$H / K$ & $\alpha_{1}^{\prime \prime \prime}+2 \alpha_{2}^{\prime \prime \prime}+3 \alpha_{3}^{\prime \prime \prime}$ & $\omega_{3}^{\prime \prime \prime}$ \\
\hline
\end{tabular}

\begin{tabular}{|lllll|}
\hline$\hat{\varphi} \quad: \quad a \omega_{1}+b \omega_{3}+c \omega_{4}$ & $\longmapsto$ & $(a+c) \omega_{1}^{\prime}+(b+c) \omega_{3}^{\prime \prime}$ \\
\hline $\bar{\varphi}: a \omega_{1}+b \omega_{3}+c \omega_{4}$ & $\longmapsto$ & $(a+b) \omega_{3}^{\prime \prime \prime}$ \\
\hline
\end{tabular}

Given $\lambda \in \Lambda_{G / K}^{+}$, recall our notations $\hat{\lambda}=\hat{\varphi}(\lambda)$ and $\bar{\lambda}=\bar{\varphi}(\lambda)$. If $\mu \in \Lambda_{G / K}^{+}$, we write $\mu \sim \lambda$ if $\hat{\mu}=\hat{\lambda}$. Identifying $\mathbb{C}[\widehat{G} / \widehat{K}] \simeq \mathbb{C}[G / K]$, by definition, we have then

$$
E_{\widehat{G} / \widehat{K}}(\hat{\lambda})=\bigoplus_{\lambda^{\prime} \sim \lambda} E_{G / K}\left(\lambda^{\prime}\right)
$$

Theorem 5.9 Let $(G, K)$ be one of the following spherical pairs of Table 3:

Sph.A6, Sph.A8, Sph.A10, Sph.A13, and Sph.A14.

Then, for all $\lambda, \mu, v \in \Lambda_{G / K}^{+}$, we have

$$
E_{G / K}(v) \subset E_{G / K}(\lambda) \cdot E_{G / K}(\mu) \Longleftrightarrow\left\{\begin{array}{c}
E_{\widehat{G} / \widehat{K}}(\hat{v}) \subset E_{\widehat{G} / \widehat{K}}(\hat{\lambda}) \cdot E_{\widehat{G} / \widehat{K}}(\hat{\mu}), \\
E_{H / K}(\bar{v}) \subset E_{H / K}(\bar{\lambda}) \cdot E_{H / K}(\bar{\mu})
\end{array}\right.
$$

Proof Suppose that $E_{G / K}(v) \subset E_{G / K}(\lambda) \cdot E_{G / K}(\mu)$. Looking at the associated $\widehat{G}$ modules generated in the coordinate ring $\mathbb{C}[\widehat{G} / \widehat{K}] \simeq \mathbb{C}[G / K]$, it follows that $E_{\widehat{G} / \widehat{K}}(\hat{v}) \subset E_{\widehat{G} / \widehat{K}}(\hat{\lambda}) \cdot E_{\widehat{G} / \widehat{K}}(\hat{\mu})$ as well. Similarly, looking at the images in $\mathbb{C}[H / K]$, we see that $E_{H / K}(\bar{v}) \subset E_{H / K}(\bar{\lambda}) \cdot E_{H / K}(\bar{\mu})$.

Suppose conversely that both the inclusions $E_{\widehat{G} / \widehat{K}}(\hat{v}) \subset E_{\widehat{G} / \widehat{K}}(\hat{\lambda}) \cdot E_{\widehat{G} / \widehat{K}}(\hat{\mu})$ and $E_{H / K}(\bar{v}) \subset E_{H / K}(\bar{\lambda}) \cdot E_{H / K}(\bar{\mu})$ hold. By the first inclusion, the restriction of functions from $G / K$ to $H / K$ induces a commutative diagram as follows:

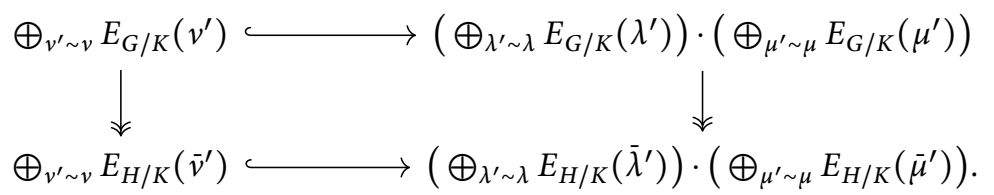

By the second inclusion, there are $\lambda^{\prime} \sim \lambda, \mu^{\prime} \sim \mu$, and $v^{\prime} \sim v$ such that $\bar{\lambda}^{\prime}=\bar{\lambda}, \bar{\mu}^{\prime}=\bar{\mu}$, $\bar{v}^{\prime}=\bar{v}$, and $E_{G / K}\left(v^{\prime}\right) \subset E_{G / K}\left(\lambda^{\prime}\right) \cdot E_{G / K}\left(\mu^{\prime}\right)$. On the other hand, by Corollary 5.7, the restriction of $\bar{\varphi}$ to the fibers of $\hat{\varphi}$ is injective. Thus, $\lambda^{\prime}=\lambda, \mu^{\prime}=\mu$, and $v^{\prime}=v$, and we get $E_{G / K}(v) \subset E_{G / K}(\lambda) \cdot E_{G / K}(\mu)$. 
Corollary 5.10 Let $(G, K)$ be one of the following spherical pairs of Table 3:

Sph.A6, Sph.A8, Sph.A10, Sph.A13, and Sph.A14.

If Conjecture 3.2 holds true, then Conjecture 5.1 holds true for $(G, K)$. In particular, Conjecture 5.1 holds true in the cases

Sph.A6, Sph.A10, Sph.A13, and Sph.A14.

Proof Set $X=G / K, Y=\widehat{G} / \widehat{K}$, and $Z=H / K$. Then, by Proposition 5.6, we have an isogeny of reductive groups

$$
G_{Y} \times G_{Z} \rightarrow G_{X}
$$

Notice that a $G_{X}$-module is irreducible if and only if it is irreducible as a $\left(G_{Y} \times G_{Z}\right)$ module under the previous map.

If $\lambda \in \Lambda_{X}^{+}$, regarding $V_{X}(\lambda)$ as a $\left(G_{Y} \times G_{Z}\right)$-module, by the definition of $\hat{\lambda}$ and $\bar{\lambda}$, we see that

$$
V_{X}(\lambda) \simeq V_{Y}(\hat{\lambda}) \otimes V_{Z}(\bar{\lambda}) .
$$

In particular, given $\lambda, \mu, v \in \Lambda_{X}^{+}$, we have

$$
V_{X}(v) \subset V_{X}(\lambda) \otimes V_{X}(\mu) \Longleftrightarrow\left\{\begin{array}{c}
V_{Y}(\hat{v}) \subset V_{Y}(\hat{\lambda}) \otimes V_{Y}(\hat{\mu}), \\
V_{Z}(\bar{v}) \subset V_{Z}(\bar{\lambda}) \otimes V_{Z}(\bar{\mu}) .
\end{array}\right.
$$

Thus, by Theorem 5.9, we see that Conjecture 5.1 holds for $X$ if and only if it holds both for $Y$ and $Z$.

If both $Y$ and $Z$ are direct products of symmetric varieties with restricted root system of type $A_{1}$, then by Stanley's Pieri rule for Jack symmetric functions, we see that Conjecture 3.2 holds true both for $Y$ and $Z$. Thus, in this case, the claim follows for $X$ as well.

Remark 5.11 There is another case which can be put in the framework outlined at the beginning of this subsection, even though $G$ is not simple. In this case,

$$
G / K=[\operatorname{Sp}(2) \times \operatorname{Sp}(2 n)] /[\operatorname{diag}(\operatorname{Sp}(2)) \times \operatorname{Sp}(2 n-2)],
$$

and $\Phi_{G / K}$ is of type $A_{1} \times A_{1}$, and Conjecture 5.1 holds true thanks to the same arguments of Theorem 5.9 and Corollary 5.10. The corresponding symmetric varieties are

$$
\begin{gathered}
\widehat{G} / \widehat{K}=\operatorname{SO}(4 n) / \operatorname{SO}(4 n-1), \\
H / K=[\operatorname{Sp}(2) \times \operatorname{Sp}(2) \times \operatorname{Sp}(2 n-2)] /[\operatorname{diag}(\operatorname{Sp}(2)) \times \operatorname{Sp}(2 n-2)] \\
\simeq[\operatorname{Sp}(2) \times \operatorname{Sp}(2)] / \operatorname{diag}(\operatorname{Sp}(2)),
\end{gathered}
$$

which have both restricted root system of type $A_{1}$. The associated spherical roots and spherical weights are described in the tables here below, together with the maps $\hat{\varphi}$ and $\bar{\varphi}$. 


\begin{tabular}{|c|c|c|}
\hline & Normalized spherical roots & Fund. spherical weights \\
\hline \hline$G / K$ & $\alpha_{0}+\alpha_{1}, \alpha_{1}+2\left(\alpha_{2}+\cdots+\alpha_{n-1}\right)+\alpha_{n}$ & $\omega_{0}+\omega_{1}, \omega_{2}$ \\
\hline$\widehat{G} / \widehat{K}$ & $2\left(\alpha_{1}^{\prime}+\cdots+\alpha_{2 n-2}^{\prime}\right)+\alpha_{2 n-1}^{\prime}+\alpha_{2 n}^{\prime}$ & $\omega_{1}^{\prime}$ \\
\hline$H / K$ & $\alpha_{0}^{\prime \prime}+\alpha_{1}^{\prime \prime}$ & $\omega_{0}^{\prime \prime}+\omega_{1}^{\prime \prime}$ \\
\hline
\end{tabular}

\begin{tabular}{|lllll|}
\hline$\hat{\varphi} \quad: \quad a\left(\omega_{0}+\omega_{1}\right)+b \omega_{2}$ & $\longmapsto$ & $(a+2 b) \omega_{1}^{\prime}$ \\
\hline $\bar{\varphi}:$ & $a\left(\omega_{0}+\omega_{1}\right)+b \omega_{2}$ & $\longmapsto$ & $a\left(\omega_{0}^{\prime \prime}+\omega_{1}^{\prime \prime}\right)$ \\
\hline
\end{tabular}

\subsection{Other cases with restricted root system of type $A$}

We now explain how the cases Sph.A7, Sph.A9, and Sph.A11 of Table 3 follow (almost immediately) from their companion cases Sph.A6, Sph.A8, and Sph.A10.

(Sph.A7) $G=\mathrm{SL}(n), K=\mathrm{GL}(n-1)$. This case is immediately reduced to Sph.A6. Denote indeed $K^{\prime}=\mathrm{SL}(n-1)$ : then $K=\mathrm{N}_{G}\left(K^{\prime}\right)$, and the spherical roots of $G / K$ are the same as those of $G / K^{\prime}$. On the other hand, $\Omega_{G / K}=\Lambda_{G / K^{\prime}}$, and $V(\lambda)^{(K)}=V(\lambda)^{K^{\prime}}$ for all $\lambda \in \Omega_{G / K}^{+}$; therefore, $\mathbb{C}[G]^{(K)}=\mathbb{C}[G]^{K^{\prime}}$.

(Sph.A9) $G=\operatorname{SL}(2 n+1), K=\mathrm{GL}(1) \times \operatorname{Sp}(2 n)$. This case is immediately reduced to Sph.A8. Consider indeed $K^{\prime}=\mathrm{Sp}(2 n)$ : then $K=\mathrm{N}_{G}\left(K^{\prime}\right)$, and the spherical roots of $G / K$ are the same as those of $G / K^{\prime}$. Moreover, as in the previous case, we have $\mathbb{C}[G]^{(K)}=\mathbb{C}[G]^{K^{\prime}}$.

(Sph.A11) $G=\operatorname{Sp}(2 n), K=\operatorname{Sp}(2) \times \operatorname{Sp}(2 n-2)$. This case is immediately reduced to Sph.A10. Set indeed $K^{\prime}=\mathrm{GL}(1) \times \mathrm{Sp}(2 n-2)$ : then $K^{\prime} \subset K$; thus, we have an inclusion $\mathbb{C}[G]^{K} \subset \mathbb{C}[G]^{K^{\prime}}$. On the other hand, the unique spherical root of $G / K$ is a spherical root of $G / K^{\prime}$ as well, and if $\lambda, \mu \in \Lambda_{G / K}$, then $\mu \leqslant_{G / K} \lambda$ if and only if $\mu \leqslant_{G / K^{\prime}} \lambda$.

\begin{tabular}{|c|c|c|}
\hline & Normalized spherical roots & Fund. sph. weights \\
\hline \hline$G / K$ & $\alpha_{1}+2\left(\alpha_{2}+\ldots+\alpha_{n-1}\right)+\alpha_{n}$ & $\omega_{2}$ \\
\hline$G / K^{\prime}$ & $\alpha_{1}, \alpha_{1}+2\left(\alpha_{2}+\ldots+\alpha_{n-1}\right)+\alpha_{n}$ & $2 \omega_{1}, \omega_{2}$ \\
\hline
\end{tabular}

As a consequence of the previous analysis, we get the following.

Proposition 5.12 Let $(G, K)$ be one of the spherical pairs Sph.A7, Sph.A9, and Sph.A11 of Table 3. If Conjecture 3.2 holds true, then Conjecture 5.1 holds true for $(G, K)$. In particular, Conjecture 5.1 holds true for Sph.A7 and Sph.A11. 


\section{A conjectural rule in the case of spherical Levi subgroups}

There is another case where we can apply the techniques of Section 5, and find two morphisms which decompose the root system of an affine spherical variety into those of two symmetric varieties. However, in this case, the root system generated by the spherical roots is of type $B$, and as far as we know, there is no rule (not even conjectural) to multiply spherical functions on a symmetric variety in this case.

\subsection{The spherical pair $(\mathrm{SO}(2 p+1), \mathrm{GL}(p))$}

Set $G=\operatorname{SO}(2 p+1)$ and $K=\mathrm{GL}(p)$ with $p \geqslant 3$. Then, the homogeneous space $G / K$ is spherical, and it is a model space for $G$ (see $[13,14,27])$.

The spherical root system $\Phi_{G / K}$ is of type $\mathrm{B}_{\lfloor p / 2\rfloor} \times \mathrm{B}_{[p / 2\rceil}$ if $p \geqslant 4$, and of type $\mathrm{A}_{1} \times$ $\mathrm{B}_{2}$ if $p=3$. In this case as well, it is possible to define reductive groups $H \subset G \subset \widehat{G}$ and two symmetric varieties $H / K$ and $\widehat{G} / \widehat{K}$ endowed with equivariant morphisms

$$
H / K \hookrightarrow G / K \stackrel{\sim}{\longrightarrow} \widehat{G} / \widehat{K},
$$

which produce an isogeny of root data such as in Proposition 5.6. This will allow to extend Theorem 5.9 to this case as well, and to reduce the multiplication of the spherical functions on $G / K$ to the case of two symmetric varieties.

More precisely, define

$$
\widehat{G}=\mathrm{SO}(2 p+2), \quad \widehat{K}=\mathrm{GL}(p+1), \quad H=\mathrm{SO}(2 p) .
$$

The root systems $\Phi_{\widehat{G} / \widehat{K}}$ and $\Phi_{H / K}$ are of type B, whereas the restricted root systems $\widetilde{\Phi}_{\widehat{G} / \widehat{K}}$ and $\widetilde{\Phi}_{H / K}$ are either of type $B C$ or of type $C$, depending on the parity of $p$.

As in Section 5, the equivariant morphisms $H / K \hookrightarrow G / K \stackrel{\sim}{\rightarrow} \widehat{G} / \widehat{K}$ induce linear maps of the respective weight monoids

$$
\hat{\varphi}: \Lambda_{G / K}^{+} \longrightarrow \Lambda_{\widehat{G} / \widehat{K}}^{+}, \quad \bar{\varphi}: \Lambda_{G / K}^{+} \longrightarrow \Lambda_{H / K}^{+} .
$$

For $\lambda \in \Lambda_{G / K}^{+}$, we set $\hat{\lambda}=\hat{\varphi}(\lambda)$ and $\bar{\lambda}=\bar{\varphi}(\lambda)$.

The spherical roots and the weight lattices, as well as the maps $\hat{\varphi}$ and $\bar{\varphi}$, are described in the tables below. We separate the two cases $p$ even and $p$ odd.

$(p=2 n$ even $)$

\begin{tabular}{|c|c|l|}
\hline & Normalized spherical roots & \multicolumn{1}{|c|}{ Fund. sph. weights } \\
\hline \hline \multirow{2}{*}{$G / K$} & $\alpha_{i}+\alpha_{i+1}(i=1, \ldots, 2 n-1), \alpha_{2 n}$ & $\begin{array}{l}\omega_{i}(i=1, \ldots, 2 n-1), \\
2 \omega_{2 n}\end{array}$ \\
\hline \multirow{G}{*}{$\widehat{K} \widehat{K}$} & $\begin{array}{l}\alpha_{2 i-1}^{\prime}+2 \alpha_{2 i}^{\prime}+\alpha_{2 i+1}^{\prime}(i=1, \ldots, n-1), \\
\alpha_{2 n-1}^{\prime}+\alpha_{2 n}^{\prime}+\alpha_{2 n+1}^{\prime}\end{array}$ & $\begin{array}{l}\omega_{2 i}^{\prime}(i=1, \ldots, n-1), \\
\omega_{2 n}^{\prime}+\omega_{2 n+1}^{\prime}\end{array}$ \\
\hline \multirow{2}{*}{$H / K$} & $\begin{array}{l}\alpha_{2 i-1}^{\prime \prime}+2 \alpha_{2 i}^{\prime \prime}+\alpha_{2 i+1}^{\prime \prime}(i=1, \ldots, n-1), \\
\alpha_{2 n}^{\prime \prime}\end{array}$ & $\begin{array}{l}\omega_{2 i}^{\prime \prime}(i=1, \ldots, n-1), \\
2 \omega_{2 n}^{\prime \prime}\end{array}$ \\
\hline
\end{tabular}




\begin{tabular}{|lllll|}
\hline$\hat{\varphi}$ & $:$ & $\sum_{i=1}^{2 n} a_{i} \omega_{i}$ & $\longmapsto$ & $\left(\sum_{i=1}^{n-1}\left(a_{2 i-1}+a_{2 i}\right) \omega_{2 i}^{\prime}\right)+\left(a_{2 n-1}+\frac{a_{2 n}}{2}\right)\left(\omega_{2 n}^{\prime}+\omega_{2 n+1}^{\prime}\right)$ \\
\hline $\bar{\varphi}$ & $:$ & $\sum_{i=1}^{2 n} a_{i} \omega_{i}$ & $\longmapsto$ & $\left(\sum_{i=1}^{n-1}\left(a_{2 i}+a_{2 i+1}\right) \omega_{2 i}^{\prime \prime}\right)+a_{2 n} \omega_{2 n}^{\prime \prime}$ \\
\hline
\end{tabular}

$(p=2 n+1$ odd $)$

\begin{tabular}{|c|c|l|}
\hline & Normalized spherical roots & Fund. sph. weights \\
\hline \hline \multirow{2}{*}{$G / K$} & $\alpha_{i}+\alpha_{i+1}(i=1, \ldots, 2 n), \alpha_{2 n+1}$ & $\omega_{i}(i=1, \ldots, 2 n)$, \\
& $\alpha_{2 n-1}^{\prime}+2 \alpha_{2 i}^{\prime}+\alpha_{2 i+1}^{\prime}(i=1, \ldots, n)$, & $\omega_{2 n+1}^{\prime}(i=1, \ldots, n)$, \\
\multirow{2}{*}{$\widehat{G} / \widehat{K}$} & $\alpha_{2 n+2}^{\prime}$ & $2 \omega_{2 n+2}^{\prime}$ \\
\hline \multirow{2}{*}{$H / K$} & $\begin{array}{c}\alpha_{2 i-1}^{\prime \prime}+2 \alpha_{2 i}^{\prime \prime}+\alpha_{2 i+1}^{\prime \prime}(i=1, \ldots, n-1), \\
\alpha_{2 n-1}^{\prime \prime}+\alpha_{2 n}^{\prime \prime}+\alpha_{2 n+1}^{\prime \prime}\end{array}$ & $\omega_{2 i}^{\prime \prime}(i=1, \ldots, n-1)$, \\
& $\omega_{2 n}^{\prime \prime}+\omega_{2 n+1}^{\prime \prime}$ \\
\hline
\end{tabular}

\begin{tabular}{|lllll|}
\hline$\hat{\varphi}$ & $:$ & $\sum_{i=1}^{2 n+1} a_{i} \omega_{i}$ & $\longmapsto$ & $\left(\sum_{i=1}^{n}\left(a_{2 i-1}+a_{2 i}\right) \omega_{2 i}^{\prime}\right)+a_{2 n+1} \omega_{2 n+2}^{\prime}$ \\
\hline $\bar{\varphi}$ & $:$ & $\sum_{i=1}^{2 n+1} a_{i} \omega_{i}$ & $\longmapsto$ & $\left(\sum_{i=1}^{n-1}\left(a_{2 i}+a_{2 i+1}\right) \omega_{2 i}^{\prime \prime}\right)+\left(a_{2 n}+\frac{a_{2 n+1}}{2}\right)\left(\omega_{2 n}^{\prime \prime}+\omega_{2 n+1}^{\prime \prime}\right)$ \\
\hline
\end{tabular}

Therefore, in this case as well, we obtain the similar results as those in Proposition 5.6 and Theorem 5.9.

Theorem 6.1 Let $(G, K)$ be the spherical pair $(\mathrm{SO}(2 p+1), \mathrm{GL}(p))$, then with the above definitions:

(i) The map

$$
(\hat{\varphi}, \bar{\varphi}): \Xi_{G / K} \longrightarrow \Xi_{\widehat{G} / \widehat{K}} \oplus \Xi_{H / K}
$$

is an isogeny of based root data

$$
\mathcal{R}_{G / K} \longrightarrow \mathcal{R}_{\widehat{G} / \widehat{K}} \oplus \mathcal{R}_{H / K}
$$

(ii) For all $\lambda, \mu, v \in \Lambda_{G / K}^{+}$, we have

$$
E_{G / K}(v) \subset E_{G / K}(\lambda) \cdot E_{G / K}(\mu) \Longleftrightarrow\left\{\begin{array}{l}
E_{\widehat{G} / \widehat{K}}(\hat{v}) \subset E_{\widehat{G} / \widehat{K}}(\hat{\lambda}) \cdot E_{\widehat{G} / \widehat{K}}(\hat{\mu}), \\
E_{H / K}(\bar{v}) \subset E_{H / K}(\bar{\lambda}) \cdot E_{H / K}(\bar{\mu}) .
\end{array}\right.
$$

The symmetric variety $\mathrm{SO}(2 n) / \mathrm{GL}(n)$, however, is not covered by Conjecture 3.2. This prompts us to wonder how the conjecture should be rephrased in this case, and more generally in the case of the symmetric varieties of Hermitian type (that is, the symmetric varieties $G / K$ with $K$ a Levi subgroup).

Notice that if $(G, K)$ is a spherical pair with $K$ a Levi subgroup of $G$, then either $G / K$ is a symmetric space of Hermitian type, or it is the pair $(\mathrm{SO}(2 p+1), \mathrm{GL}(p))$ considered above, or it is the pair $(\mathrm{Sp}(2 p), \mathrm{GL}(1) \times \mathrm{Sp}(2 p-2))$ which appeared as case Sph.A10 in Section 5.2. In particular, the analysis in this section reduces the case of the spherical Levi subgroups to the case of the Hermitian symmetric subgroups. 


\subsection{The Hermitian symmetric case}

Let $X=G / K$ be a symmetric variety of Hermitian type, with $G$ simple and simply connected. This means that the center of $K$ has positive dimension, or equivalently that $K$ is a Levi subgroup of $G$. In this case, the restricted root system $\widetilde{\Phi}_{X}$ is either of type $C$ or $B C$, whereas the root system $\Phi_{X}$ is always of type $B$.

For completeness, we list in Table 4 all the symmetric varieties of Hermitian type. In all cases, we also give the simple restricted roots and a minimal set of generators for the weight monoid $\Lambda_{X}^{+}$.

(i) $\mathrm{SL}(\mathrm{p}+\mathrm{q}) / \mathrm{S}(\mathrm{GL}(\mathrm{p}) L \times \mathrm{GL}(\mathrm{q})), \quad q>p \geqslant 1: \quad \Delta_{X}=\left\{\alpha_{i}+\alpha_{p+q-i}: \quad 1 \leqslant i<\right.$ $p\} \cup\left\{\alpha_{p}+\cdots+\alpha_{q}\right\}, \Lambda_{X}^{+}$is generated by the weights $\omega_{i}+\omega_{p+q-i}$ with $1 \leqslant i \leqslant p$.

(ii) $\mathrm{SL}(2 \mathrm{p}) / \mathrm{S}(\mathrm{GL}(\mathrm{p}) \times \mathrm{GL}(\mathrm{p})), p \geqslant 1: \Delta_{X}=\left\{\alpha_{i}+\alpha_{2 p-i}: 1 \leqslant i<p\right\} \cup\left\{\alpha_{p}\right\}, \Lambda_{X}^{+}$is generated by the weights $\omega_{i}+\omega_{2 p-i}$ with $1 \leqslant i<p$, together with $2 \omega_{p}$.

(iii) $\operatorname{Spin}(2 \mathrm{p}+1) / \operatorname{Spin}(2) \times \operatorname{Spin}(2 \mathrm{p}-1), \quad p \geqslant 2: \Delta_{X}=\left\{\alpha_{1}, 2\left(\alpha_{2}+\cdots+\alpha_{p}\right)\right\}, \Lambda_{X}^{+}$is generated by $2 \omega_{1}$ and $\omega_{2}$.

(iv) $\operatorname{Spin}(2 \mathrm{p}) / \operatorname{Spin}(2) \times \operatorname{Spin}(2 \mathrm{p}-2), \quad p \geqslant 3: \quad \Delta_{X}=\left\{\alpha_{1}, 2\left(\alpha_{2}+\cdots+\alpha_{p-2}\right)+\alpha_{p-1}+\right.$ $\left.\alpha_{p}\right\}, \Lambda_{X}^{+}$is generated by $2 \omega_{1}$ and $\omega_{2}$.

(v) $\mathrm{Sp}(2 \mathrm{p}) / \mathrm{GL}(\mathrm{p}), p \geqslant 2: \Delta_{X}=\left\{2 \alpha_{i}: 1 \leqslant i \leqslant p-1\right\} \cup\left\{\alpha_{p}\right\}, \Lambda_{X}^{+}$is generated by the weights $2 \omega_{i}$ with $1 \leqslant i \leqslant p$.

(vi) $\operatorname{Spin}(4 \mathrm{p}+2) / \mathrm{GL}(2 \mathrm{p}+1), \quad p \geqslant 1: \quad \Delta_{X}=\left\{\alpha_{2 i-1}+2 \alpha_{2 i}+\alpha_{2 i+1}: 1 \leqslant i<p\right\} \cup$ $\left\{\alpha_{2 p-1}+\alpha_{2 p}+\alpha_{2 p+1}\right\}, \Lambda_{X}^{+}$is generated by the weights $\omega_{2 i}$ with $1 \leqslant i<p$ together with $\omega_{2 p}+\omega_{2 p+1}$.

(vii) Spin(4p)/GL(2p), $p \geqslant 2: \Delta_{X}=\left\{\alpha_{2 i-1}+2 \alpha_{2 i}+\alpha_{2 i+1}: 1 \leqslant i \leqslant p-1\right\} \cup\left\{\alpha_{2 p}\right\}$, $\Lambda_{X}^{+}$is generated by the weights $\omega_{2 i}$ with $1 \leqslant i<p$, together with $2 \omega_{2 p}$.

(viii) $\mathrm{E}_{6} / \mathrm{D}_{5} \times \mathbb{C}^{*}: \quad \Delta_{X}=\left\{\alpha_{1}+\alpha_{3}+\alpha_{4}+\alpha_{5}+\alpha_{6}, 2 \alpha_{2}+\alpha_{3}+2 \alpha_{4}+\alpha_{5}\right\}, \quad \Lambda_{X}^{+} \quad$ is generated by $\omega_{1}+\omega_{6}$ and $\omega_{2}$.

(ix) $\mathrm{E}_{7} / \mathrm{E}_{6} \times \mathbb{C}^{*}: \quad \Delta_{X}=\left\{2 \alpha_{1}+\alpha_{2}+2 \alpha_{3}+2 \alpha_{4}+\alpha_{5}, \alpha_{2}+\alpha_{3}+2\left(\alpha_{4}+\alpha_{5}+\right.\right.$ $\left.\left.\alpha_{6}\right), \alpha_{7}\right\}, \Lambda_{X}^{+}$is generated by $\omega_{1}, \omega_{6}$, and $2 \omega_{7}$.

If $\widetilde{\Phi}_{X}$ is nonreduced, then $K$ is self-normalizing, and the derived subgroup $K^{\prime}$ is also spherical in $G$. If instead $\widetilde{\Phi}_{X}$ is reduced, then $K$ is not self-normalizing, and its derived subgroup is not spherical in $G$. Therefore, there are some Hermitian symmetric subgroups which have a nonsymmetric semisimple spherical counterpart, similarly to the cases Sph.A7 and Sph.A9 of Table 3.

We list such spherical pairs in Table 5. In all cases, we also give the simple restricted roots and a minimal set of generators for the weight monoid $\Lambda_{X}^{+}$.

(i) $\operatorname{SL}(\mathrm{p}+\mathrm{q}) / \mathrm{SL}(\mathrm{p}) \times \mathrm{SL}(\mathrm{q})), q>p \geqslant 1: \Delta_{X}=\left\{\alpha_{i}+\alpha_{p+q-i}: 1 \leqslant i<p\right\} \cup\left\{\alpha_{p}+\right.$ $\left.\cdots+\alpha_{q}\right\}, \Lambda_{X}^{+}$is generated by the weights $\omega_{i}+\omega_{p+q-i}$ with $1 \leqslant i<p$ together with $\omega_{p}$ and $\omega_{q}$.

(vi') $\operatorname{Spin}(4 \mathrm{p}+2) / \mathrm{SL}(2 \mathrm{p}+1), \quad p \geqslant 1: \quad \Delta_{X}=\left\{\alpha_{2 i-1}+2 \alpha_{2 i}+\alpha_{2 i+1} \quad: \quad 1 \leqslant i<\right.$ $p\} \cup\left\{\alpha_{2 p-1}+\alpha_{2 p}+\alpha_{2 p+1}\right\}, \Lambda_{X}^{+}$is generated by the weights $\omega_{2 i}$ with $1 \leqslant i<p$ together with $\omega_{2 p}$ and $\omega_{2 p+1}$.

(viii') $\mathrm{E}_{6} / \mathrm{D}_{5}: \Delta_{X}=\left\{\alpha_{1}+\alpha_{3}+\alpha_{4}+\alpha_{5}+\alpha_{6}, 2 \alpha_{2}+\alpha_{3}+2 \alpha_{4}+\alpha_{5}\right\}, \Lambda_{X}^{+}$is generated by $\omega_{1}, \omega_{2}$, and $\omega_{6}$.

If $\left(G, K^{\prime}\right)$ is a spherical pair appearing in Table 5 and $K=\mathrm{N}_{G}\left(K^{\prime}\right)$ is the corresponding symmetric subgroup of Hermitian type, then we have $\Omega_{G / K}^{+}=\Lambda_{G / K^{\prime}}^{+}$ 


\begin{tabular}{ccccc}
\hline & $G$ & $K$ & $\widetilde{\Phi}_{G / K}$ & $\Phi_{G / K}$ \\
\hline He.1a & $\mathrm{SL}(p+q), q>p \geqslant 1$ & $\mathrm{~S}(\mathrm{GL}(p) \times \mathrm{GL}(q))$ & $\mathrm{BC}_{p}$ & $\mathrm{~B}_{p}$ \\
He.1b & $\mathrm{SL}(2 p), p \geqslant 1$ & $\mathrm{~S}(\mathrm{GL}(p) \times \mathrm{GL}(p))$ & $\mathrm{C}_{p}$ & $\mathrm{~B}_{p}$ \\
He.2 & $\mathrm{Spin}(n), n \geqslant 5$ & $\mathrm{Spin}(2) \times \mathrm{Spin}(n-2)$ & $\mathrm{C}_{2}$ & $\mathrm{~B}_{2}$ \\
He.3 & $\mathrm{Sp}(2 p), p \geqslant 3$ & $\mathrm{GL}(p)$ & $\mathrm{C}_{p}$ & $\mathrm{~B}_{p}$ \\
He.4a & $\mathrm{Spin}(4 p+2), p \geqslant 2$ & $\mathrm{GL}(2 p+1)$ & $\mathrm{BC}_{p}$ & $\mathrm{~B}_{p}$ \\
He.4b & $\mathrm{Spin}(4 p), p \geqslant 3$ & $\mathrm{GL}(2 p)$ & $\mathrm{C}_{p}$ & $\mathrm{~B}_{p}$ \\
He.5 & $\mathrm{E}_{6}$ & $\mathrm{D}_{5} \times \mathbb{C}^{*}$ & $\mathrm{BC}_{2}$ & $\mathrm{~B}_{2}$ \\
He.6 & $\mathrm{E}_{7}$ & $\mathrm{E}_{6} \times \mathbb{C}^{*}$ & $\mathrm{C}_{3}$ & $\mathrm{~B}_{3}$ \\
\hline
\end{tabular}

Table 4: Symmetric varieties of Hermitian type.

\begin{tabular}{cccc}
\hline & $G$ & $K$ & $\Phi_{G / K}$ \\
\hline He.1a' & $\operatorname{SL}(p+q), q>p \geqslant 1$ & $\mathrm{SL}(p) \times \operatorname{SL}(q)$ & $\mathrm{B}_{p}$ \\
He.4a' & $\operatorname{Spin}(4 p+2), p \geqslant 1$ & $\mathrm{SL}(2 p+1)$ & $\mathrm{B}_{p}$ \\
He.5’ & $\mathrm{E}_{6}$ & $\mathrm{D}_{5}$ & $\mathrm{~B}_{2}$ \\
\hline
\end{tabular}

Table 5: Nonsymmetric reductive spherical pairs $(G, K)$ with $G / \mathrm{N}_{G}(K)$ symmetric of Hermitian type.

and $\mathbb{C}[G]^{(K)}=\mathbb{C}[G]^{K^{\prime}}$, as in the cases Sph.A7 and Sph.A9. Thus, the problem of decomposing the product of two spherical functions on $G / K$ reduces to the analogous problem on $G / K^{\prime}$.

In the same spirit of Conjectures 3.2 and 5.1, we formulate the following for the multiplication of spherical functions in terms of the tensor product of the restricted root system, which by computational experiments seems to hold for all the Hermitian symmetric pairs but in case He.la.

Conjecture 6.2 Let $(G, K)$ be a reductive spherical pair with $G$ simple and $K$ a Levi subgroup of $G$ different from case He.1a. For all $\lambda, \mu, v \in \Lambda_{X}^{+}$, it holds

$$
E_{G / K}(v) \subset E_{G / K}(\lambda) \cdot E_{G / K}(\mu) \Longleftrightarrow\left\{\begin{array}{c}
V_{X}(v) \subset V_{X}(\lambda) \otimes V_{X}(\mu), \\
v \leqslant_{X} \lambda+\mu .
\end{array}\right.
$$

By Theorem 6.1 and Corollary 5.10, the previous conjecture immediately reduces to the case where $G / K$ is a symmetric variety of Hermitian type. 
Remark 6.3 In [4, Section 9], the following question was considered. Suppose that $G$ is simply laced, and let $X=G / K$ be a spherical variety with $\Delta_{X}^{\text {sc }} \cap \Delta=\varnothing$. Let $\lambda, \mu, v \in$ $\Lambda_{X}^{+}$, is it true that $E_{X}(v) \subset E_{X}(\lambda) \cdot E_{X}(\mu)$ if and only if $V(v) \subset V(\lambda) \otimes V(\mu)$ and $v \leqslant \Delta_{x}^{\text {sc }} \lambda+\mu$ ?

This indeed seems to be true in several cases, and in particular in case He.la. Assuming the validity of Conjecture 3.2, it is easy to see that when $X$ is symmetric and both $\Phi$ and $\Phi_{X}$ are of type A, a positive answer to the previous question follows from the saturation theorem [22]. See also [16], where a similar question is considered for the symmetric varieties with restricted root system of type A. However, the following example shows that the previous question does not always have a positive answer, at least when $\widetilde{\Phi}_{X}$ is nonreduced. Conjecture 6.2 is a partial attempt to fix the question.

Example 6.4 Consider the symmetric variety $X=\operatorname{Spin}(10) / \mathrm{GL}(5)$. The restricted root system $\widetilde{\Phi}_{X}$ is of type $B C_{2}$, with base

$$
\widetilde{\Delta}_{X}=\left\{\alpha_{1}+2 \alpha_{2}+\alpha_{3}, \alpha_{3}+\alpha_{4}+\alpha_{5}\right\} .
$$

Let us consider the highest root $\theta=\alpha_{1}+2 \alpha_{2}+2 \alpha_{3}+\alpha_{4}+\alpha_{5}$ : then $\theta \in \Lambda_{X}^{+}$and $\theta \leqslant_{X}$ $2 \theta$. Moreover, $V(\theta) \subset V(\theta)^{\otimes 2}$; however, taking the product inside $\mathbb{C}[X]$, we have $E_{X}(\theta) \notin E_{X}(\theta)^{2}$ : indeed, the unique nonzero equivariant projection

$$
\pi: V(\theta) \otimes V(\theta) \rightarrow V(\theta)
$$

is given by the bracket on the Lie algebra $\mathfrak{g} \simeq V(\theta)$. It follows that $\pi(v \otimes v)=0$, for all $v \in V(\theta)$, and we conclude by Proposition 2.2.

The same happens more generally for $\operatorname{Spin}(2 p) / \mathrm{GL}(p)$ whenever $p$ is odd, and for $\mathrm{E}_{6} / \mathrm{D}_{5} \times \mathbb{C}^{*}$ : indeed, in all these cases, the highest root is a spherical weight, and $V(\theta)$ occurs in $V(\theta)^{\otimes 2}$ with multiplicity 1 .

Both these examples are instances of exceptional symmetric varieties of Hermitian type, that is, symmetric varieties of Hermitian type with nonreduced restricted root system. There is another instance of such a symmetric variety, namely $X=\operatorname{SL}(p+$ $q) / \mathrm{S}(\mathrm{GL}(p) \times \mathrm{GL}(q))$ when $p \neq q$ : however, in this case, $V(\theta)$ occurs in $V(\theta)^{\otimes 2}$ with multiplicity 2 , and the inclusion $E_{X}(\theta) \subset E_{X}(\theta)^{2}$ holds true inside $\mathbb{C}[X]$.

Acknowledgment We would like to thank the anonymous referees for useful comments.

\section{References}

[1] D. Akhiezer and D. Panyushev, Multiplicities in the branching rules and the complexity of homogeneous spaces. Mosc. Math. J. 2(2002), 17-33.

[2] R. Avdeev and S. Cupit-Foutou, New and old results on spherical varieties via moduli theory. Adv. Math. 328(2018), 1299-1352.

[3] R. J. Beerends and E. M. Opdam, Certain hypergeometric series related to the root system BC. Trans. Amer. Math. Soc. 339(1993), 581-609.

[4] P. Bravi, J. Gandini, and A. Maffei, Projective normality of model varieties and related results. Represent. Theory 20(2016), 39-93.

[5] P. Bravi and D. Luna, An introduction to wonderful varieties with many examples of type $F_{4}$. J. Algebra 329(2011), 4-51. 
[6] P. Bravi and G. Pezzini, The spherical systems of the wonderful reductive subgroups. J. Lie Theory 25(2015), 105-123.

[7] M. Brion, Vers une généralisation des espaces symétriques. J. Algebra 134(1990), 115-143.

[8] M. Brion and F. Pauer, Valuations des espaces homogènes sphériques. Comment. Math. Helv. 62(1987), 265-285.

[9] R. Chirivì, P. Littelmann, and A. Maffei, Equations defining symmetric varieties and affine Grassmannians. Int. Math. Res. Not. IMRN 2009(2009), 291-347.

[10] C. De Concini, Normality and non normality of certain semigroups and orbit closures. In: Algebraic transformation groups and algebraic varieties, (edited by V. L. Popov) Enc. Math. Sci., 132, Springer, Berlin, 2004, pp. 15-35.

[11] C. De Concini and C. Procesi, Complete symmetric varieties. In: Invariant theory, (edited by F. Gherardelli) Lect. Notes in Math., 996, Springer, 1983, pp. 1-44.

[12] J. Gandini, Spherical orbit closures in simple projective spaces and their normalizations. Transform. Groups 16(2011), 109-136.

[13] I. M. Gelfand and A. V. Zelevinsky, Models of representations of classical groups and their hidden symmetries. Funktsional. Anal. i Prilozhen. 18(1984), no. 3, 14-31.

[14] I. M. Gelfand and A. V. Zelevinsky, Representation models for classical groups and their higher symmetries, in: Élie Cartan et les mathématiques d'aujourd'hui, Astérisque, Numero Hors Serie, 1985, pp. 117-128

[15] V. V. Gorbatsevich and A. L. Onishchik, Lie transformation groups. In: Lie groups and Lie algebras I. (edited by A. L. Onishchik), Enc. Math. Sci., 20, Springer, Berlin-Heidelberg, 1993, pp. 95-229.

[16] W. Graham and M. Hunziker, Multiplication of polynomials on Hermitian symmetric spaces and Littlewood-Richardson coefficients. Canad. J. Math. 61(2009), 351-372.

[17] G. J. Heckman and E. M. Opdam, Jacobi polynomials and hypergeometric functions associated with root systems. In: Encyclopedia of special functions: the Askey-Bateman project. Volume II: multivariable special functions, (edited by H. Koornwinder and J. V. Cambridge University Press, Cambridge, 2020, pp. 217-257.

[18] F. Knop, Automorphisms, root systems, and compactifications of homogeneous varieties. J. Amer. Math. Soc. 9(1996), 153-174.

[19] F. Knop and G. Röhrle, Spherical subgroups in simple algebraic groups. Compositio Math. 151(2015), 1288-1308.

[20] F. Knop and S. Sahi, A recursion and a combinatorial formula for Jack polynomials. Invent. Math. 128(1997), 9-22.

[21] F. Knop and B. Schalke, The dual group of a spherical variety. Trans. Moscow Math. Soc. 78(2017), 187-216.

[22] A. Knutson and T. Tao, The honeycomb model of $G L_{n}(\mathbb{C})$ tensor products. I: Proof of the saturation conjecture. J. Amer. Math. Soc. 12(1999), 1055-1090.

[23] M. Krämer, Sphärische Untergruppen in kompakten zusammenhängenden Liegruppen. Compositio Math. 38(1979), 129-153.

[24] M. W. Liebeck, J. Saxl, and G. M. Seitz, Factorizations of simple algebraic groups. Trans. Amer. Math. Soc. 348(1996), 799-822.

[25] D. Luna, Sur les orbites fermées des groupes algébriques réductifs. Invent. Math. 16(1972), 1-5.

[26] D. Luna, Variétés sphériques de type A. Publ. Math. Inst. Hautes Études Sci. 94(2001), 161-226.

[27] D. Luna, La variété magnifique modèle. J. Algebra 313(2007), 292-319.

[28] I. G. Macdonald, Symmetric functions and Hall polynomials. 2nd ed., Oxford University Press, New York, 1995.

[29] A. L. Onishchik, Inclusion relations among transitive compact transformation groups. Trudy Mosk. Mat. Obshch. 11(1962), 199-242, English transl.: Amer. Math. Soc. Transl. (2) 50(1966), 5-58.

[30] R. W. Richardson, Orbits, invariants, and representations associated to involutions of reductive groups. Invent. Math. 66(1982), 287-312.

[31] G. C. M. Ruitenburg, Invariant ideals of polynomial algebras with multiplicity free group action. Compositio Math. 71(1989), 181-227.

[32] H. Schlichtkrull, P. E. Trapa, and D. A. Vogan, Laplacians on spheres. Sao Paulo J. Math. Sci. 12(2018), 295-358.

[33] R. P. Stanley, Some combinatorial properties of Jack symmetric functions. Adv. Math. 77(1989), 76-115.

[34] E. B. Vinberg and B. N. Kimelfeld, Homogeneous domains on flag manifolds and spherical subgroups of semisimple Lie groups. Funct. Anal. Appl. 12(1978), 168-174.

[35] T. Vust, Opérations de groupes réductifs dans un type de cônes presque homogènes. Bulletin de la $\mathrm{S}$. M. F. 102(1974), 317-333. 
[36] T. Vust, Plongements d'espaces symétriques algébriques: une classification. Ann. Scuola Norm. Sup. Pisa Cl. Sci. 17(1990), no. 4, 165-195.

Dipartimento di Matematica, Sapienza Università di Roma, Piazzale A. Moro 2, 00185 Roma, Italy e-mail: bravi@mat.uniroma1.it

Dipartimento di Matematica, Università di Bologna, Piazza di Porta San Donato 5, 40126 Bologna, Italy e-mail: jacopo.gandini@unibo.it 